\title{
Observer-dependent black hole interior from operator collision
}

\author{
Beni Yoshida $\odot$ \\ Perimeter Institute for Theoretical Physics, Waterloo, Ontario N2L 2Y5, Canada
}

(Received 6 April 2020; accepted 8 January 2021; published 5 February 2021)

\begin{abstract}
We present concrete construction of interior operators for a black hole which is perturbed by an infalling observer. The construction is independent from the initial states of the black hole while dependent only on the quantum state of the infalling observer. The construction has a natural interpretation from the perspective of the boundary operator's growth, resulting from the collision between operators accounting for the infalling and outgoing modes. The interior partner modes are created once the infalling observer measures the outgoing mode, suggesting that the black hole interior is observer dependent. Implications of our results on various conceptual puzzles, including the firewall puzzle, the Marolf-Wall puzzle, and the information problem, are discussed. Explicit counterarguments against the ER $=$ EPR approach by Maldacena and Susskind are also presented.
\end{abstract}

DOI: 10.1103/PhysRevD.103.046004

\section{INTRODUCTION}

In the last decade, our understanding of the black hole interior has significantly advanced by using ideas from quantum information theory. It has led to a conjecture that bulk operators within the entanglement wedge enclosed by the extremal surface of minimal area can be reconstructed on boundary degrees of freedom in the AdS/CFT correspondence [1-4]. The attempt to find concrete expressions of bulk operators, however, has been only partially successful. While bulk operators in the smaller causal wedge were explicitly constructed [5], those outside the causal wedge remain elusive. And quite unfortunately black hole interior operators are the prime example of those behind the causal horizon. See a review [6] for a more complete list of references on this subject.

Recently, we pointed out that black hole interior operators can be constructed in boundary degrees of freedom by a procedure similar to the Hayden-Preskill thought experiment that runs backward in time [7,8]. We envision that this observation is crucial in developing a generic prescription for reconstructing bulk operators behind the causal wedge and will provide important insights on the physics of the black hole interior. The goal of the present paper is to write down concrete expressions of interior operators by explicitly implementing the ideas outlined in these works. We also discuss their physical interpretations and implications to various conceptual puzzles concerning black hole interiors.

Published by the American Physical Society under the terms of the Creative Commons Attribution 4.0 International license. Further distribution of this work must maintain attribution to the author(s) and the published article's title, journal citation, and DOI. Funded by SCOAP ${ }^{3}$.
The key challenge is to understand the experience of the infalling observer by examining the validity of semiclassical bulk descriptions properly. What distinguishes our approach from many of previous ones is to include the infalling observer explicitly as a part of the quantum system (Fig. 1). Adding an infalling observer has a rather dramatic effect which disentangles the outgoing mode from the early radiation completely. This conclusion follows from a certain quantum information theoretic relation, referred to as the decoupling theorem. It asserts separability of two subsystems due to scrambling dynamics. Hence, the preexisting entanglement plays no role in describing the infalling observer's experience. This is of course a counterargument against the "ER = EPR approaches" to the firewall puzzle [9]. ${ }^{1}$

The most salient feature of our construction is that it does not involve any degrees of freedom from the early radiation and hence is independent of initial states. This is a direct consequence of the aforementioned decoupling theorem. It is however important to recognize that the construction still depends on how the infalling observer is introduced to the system and hence is observer dependent. Observer dependence of the black hole interior is reflected in the fact

\footnotetext{
${ }^{1}$ The $\mathrm{ER}=\mathrm{EPR}$ slogan refers to the idea of identifying quantum entanglement as spacetime. Here, ER refers to the Einstein-Rosen bridge and EPR refers to the Einstein-PodolskyRosen pair. On the other hand, the ER $=$ EPR approach does not refer to the idea of identifying quantum entanglement as a wormhole. It refers to a specific proposal for resolution of the firewall puzzle by Maldacena and Susskind speculated in [9] as well as a similar resolution due to Raamsdonk [10]. In this paper, we will essentially argue that the $\mathrm{ER}=\mathrm{EPR}$ approach is incorrect. On the other hand, there is nothing incorrect about the idea of identifying quantum entanglement as a wormhole.
} 


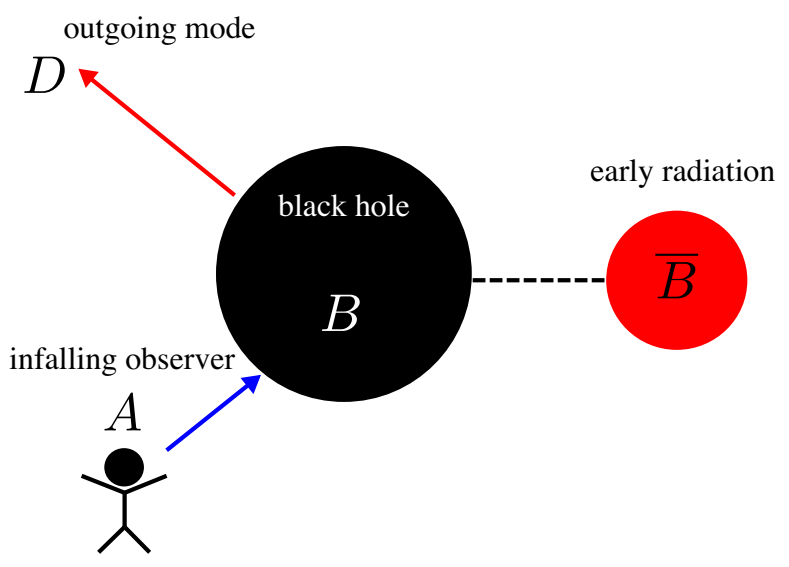

FIG. 1. A cartoon picture of the problem setting. A black hole is perturbed by an infalling observer. Our goal is to construct the interior partner operator of the outgoing mode.

that expressions of interior modes are not unique, a manifestation of its quantum error-correction property. ${ }^{2}$ We will see that observer dependence provides resolutions and useful insights on various conceptual puzzles concerning black hole interior, including the firewall puzzle, the Page curve, and the Marolf-Wall puzzle (concerning whether two observers can meet inside a two-sided black hole or not).

Our construction bears some similarity with a pioneering work by Verlinde and Verlinde, written just 4 months after the AMPS (Almheiri-Marolf-Polchinski-Sully) [12] paper, which utilized the idea of quantum error correction to construct interior operators [13]. The new ingredient in our work is to relate reconstructability to the concept of scrambling as diagnosed by out-of-time order correlators (OTOCs). Indeed, the construction is closely related to the boundary operator's growth in the AdS/CFT correspondence [14]. We will see that the interior partner of the outgoing operator $W$ can be constructed by time evolving the infalling operator $V$ and looking at the overlap with $W$. This suggests that the interior modes are created once the outgoing modes are measured by the infalling observer, highlighting its observer dependence.

Throughout the main text of the paper, we will use the infinite temperature approximation which treats a quantum black hole as an $n$-qubit quantum state where $S_{\mathrm{BH}}=n$ is the Bekenstein-Hawking coarse-grained entropy. Generalization to finite temperature systems is discussed in the Appendix D. Unitarity and scrambling dynamics are assumed in this paper. We assume tensor factorization into subsystems for the Hilbert space, but we believe that this is not a crucial limitation of our argument.

\footnotetext{
${ }^{2}$ It is perfectly fine to construct interior operators in a statedependent manner by using the early radiation as in [11], for instance. However, the decoupling theorem suggests that such construction is not relevant to the experience of the infalling observer when the backreaction is taken into account.
}

This paper is organized as follows. In Sec. II, we present the problem setting and the decoupling theorem. In Sec. III, we obtain concrete expressions of interior partner operators. In Sec. IV, we relate the construction to the operator growth. In Sec. V, we discuss observer dependence. In Sec. VI, we discuss implications of our construction to conceptual puzzles. In Sec. VII, we provide counterarguments against some of previous approaches. In Sec. VIII, we discuss the cases where the black hole does not satisfy underlying assumptions of the decoupling theorem. In Appendix A, we present the definition of scrambling and summarize diagrammatic tensor notations. In Appendix B, we present a quantitative version of the decoupling theorem in terms of the $L^{1}$ distance. In Appendix C, we discuss the thought experiment by AMPS. In Appendix D, we obtain expressions of interior partner operators at finite temperature by imposing one technical assumption. In Appendix E, a certain calculation of OTOCs is presented. In Appendix F, we justify the finite temperature assumption.

\section{BLACK HOLE DECOUPLING THEOREM}

In this section, we present the problem setup which explicitly includes the infalling observer as a part of the dynamics of the black hole. We then provide a precise definition of interior partner operators in our setup. Finally, we present the black hole decoupling theorem which suggests separability (decoupling) of the outgoing Hawking mode and the early radiation when an infalling observer is present. A summary of our notation is given in Fig. 2 for convenience.

The main result of this section is a mathematically rigorous proof that the interior partner operator can be constructed in a state-independent manner when the black hole's dynamics is scrambling. An explicit construction will be presented in the next section. While we will phrase the black hole decoupling theorem by using the black hole physics language, the theorem itself is a rigorous quantum information theoretic statement applicable to generic scrambling systems. Our strategy in this paper is to carefully deduce the bulk semiclassical description (and its possible breakdown) by relying on predictions from

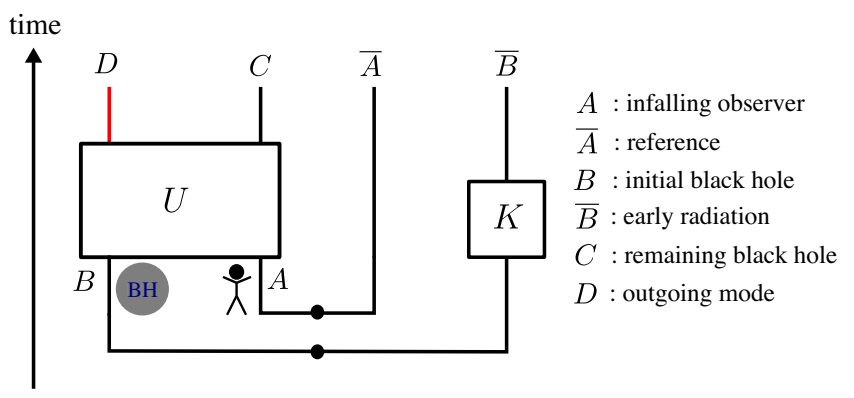

FIG. 2. A summary of the notation. Horizontal lines with dots represent EPR pairs while $K$ is an arbitrary unitary. 
rigorous quantum information theoretic results on boundary quantum mechanical systems.

\section{A. Black hole with infalling observer}

We will be primarily interested in a black hole which has emitted more than a half of its entropy content. Such an old black hole is maximally entangled with the early radiation that has been already emitted. Here we simplify the problem by expressing the black hole as a discrete system of $S_{\mathrm{BH}}=\log _{2} d$ qubits where $S_{\mathrm{BH}}$ is the BekensteinHawking coarse-grained entropy.

Then, the initial state of the old black hole can be chosen as a generic maximally entangled state between the black hole $B$ and the early radiation $\bar{B}$. Recall that a maximally entangled state can be represented as

$$
(I \otimes K)|\mathrm{EPR}\rangle_{B \bar{B}} \quad|\mathrm{EPR}\rangle_{B \bar{B}} \equiv \frac{1}{\sqrt{d_{B}}} \sum_{j=1}^{d_{B}}|j\rangle_{B} \otimes|j\rangle_{\bar{B}},
$$

where $K$ is an arbitrary unitary operator acting on the early radiation $\bar{B}$. In general, the Hilbert space for the radiation $\bar{B}$ is larger than $B$ (and is possibly unbounded). Here we think

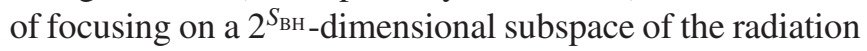
Hilbert space which is entangled with the black hole $B$.
Hence, these "qubits" on $B$ and $\bar{B}$ should be thought of as coarse-grained degrees of freedom. ${ }^{3}$

Imagine that an infalling observer (or some measurement apparatus) $A$ is dropped toward the black hole at time $t=0$ (Fig. 2). The ultimate goal of this research program is to probe the degrees of freedom behind the black hole horizon. A much more modest, yet still important, goal here is to examine the effect of including $A$ by relying on rigorous quantum information theoretic concepts in an unambiguous manner. Depending on initial states of the infalling observer $A$, the black hole will respond in different ways. Rather than keeping track of the outcomes for all the possible input states on $A$, it is convenient to append a reference system $\bar{A}$ which is entangled with the probe $A$ and forming EPR pairs $|\mathrm{EPR}\rangle_{A \bar{A}}=\frac{1}{\sqrt{d_{A}}} \sum_{j}|j\rangle_{A} \otimes|j\rangle_{\bar{A}}$. Being entangled with $A$, the reference $\bar{A}$ effectively keeps track of different input quantum states on $A$; if $\bar{A}$ is projected onto $\left|\psi^{*}\right\rangle$ (a complex conjugate of $|\psi\rangle$ ), $A$ is set to $|\psi\rangle$. The use of entangled reference systems is a standard technique in quantum information and communication theory, and often used in black hole physics, dating back to the work by Hayden and Preskill [15].

After the time evolution by scrambling unitary dynamics $U=U(t)$, the system evolves to

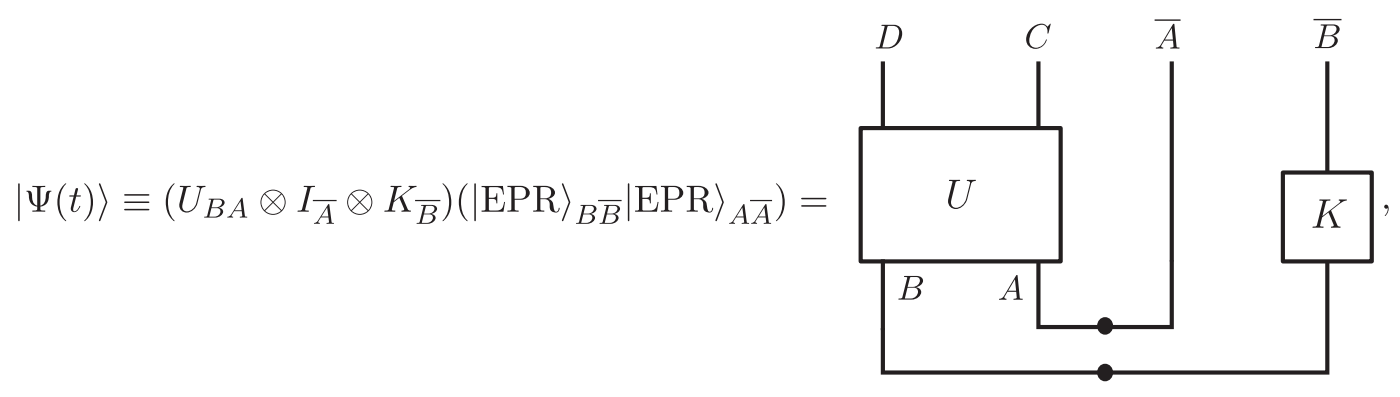

where $D$ is the outgoing mode and $C$ is the remaining black hole. Here black dots represent factor of $1 / \sqrt{d_{R}}$ in a subsystem $R$ for proper normalization of $|\mathrm{EPR}\rangle_{R \bar{R}}$. A precise definition of scrambling unitary $U$ is presented in Eq. (A2) in Appendix A. The unitary operator $U$ is defined from $A B$ to $C D$ and we assume $d_{A} d_{B}=d_{C} d_{D}$. We also assumed that the black hole Hilbert space factorizes into $\mathcal{H}_{A} \otimes \mathcal{H}_{B} \simeq \mathcal{H}_{C} \otimes \mathcal{H}_{D}$ for simplicity of discussion. This is not an essential restriction of our treatment as the decoupling phenomena occur for systems with nonfactorizable Hilbert space too; see [7] for instance.

The physics we hope to capture is as follows. An infalling observer $A$ jumps into the black hole from an

\footnotetext{
${ }^{3}$ In the end, we will prove the decoupling from the early radiation $\bar{B}$, so the finiteness of $\bar{B}$ is not an essential assumption. A more crucial assumption is the finiteness of the black hole Hilbert space $B$.
}

asymptotic anti-de Sitter (AdS) boundary (or, somewhere sufficiently away from the black hole horizon). We assume that the observer $A$ simply falls into the black hole and does not perform any particular measurement during her fall; she just attempts to cross the black hole horizon via free fall. After time $t$ (which is longer than the scrambling time, as measured in the boundary clock), the outgoing mode $D$ reaches the AdS boundary while, in the intermediate time, $A$ and $D$ would have encountered with each other near the black hole horizon [15]. Even if $A$ and $D$ do not interact directly with each other in their local frames, their encounter necessarily introduces an indirect interaction mediated by gravitational backreaction. Namely, no matter how small the infalling observer $A$ is, its effect is amplified due to the blueshift (relative to the $t=0$ slice) and creates a gravitational shockwave when $t$ becomes order of the scrambling time [16]. Our goal is to examine the effect of such gravitational interactions on the 
entanglement structure of the black hole. It is worth emphasizing that what we find in this section must be a universal property of gravitational systems with a black hole horizon.

Now we are interested in constructing interior operators for this perturbed black hole state $|\Psi(t)\rangle$. For an arbitrary operator $O_{D}$ acting on the outgoing mode, interior partner

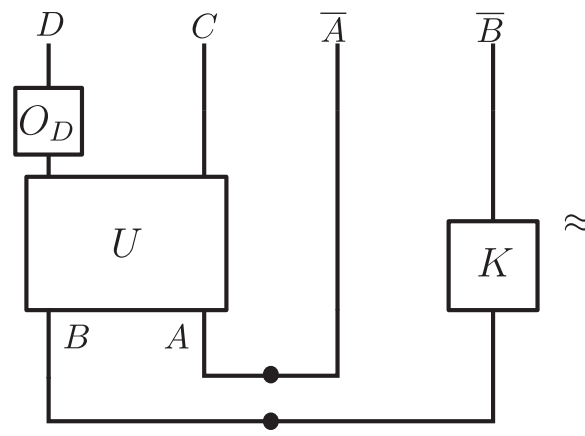

Here " $\approx$ " in Eq. (3) is evaluated by the overlap (fidelity) between the lhs and rhs.

The existence of a partner operator $\Omega\left(O_{D}\right)$ is guaranteed since the mutual information $I(D, C \overline{A B})$ is maximal, i.e., $D$ is maximally entangled with $C \overline{A B}$. One possible form of partner operators can be immediately constructed on $\overline{A B}$ by evolving $O_{D}$ backward in time and using the following relation:

$$
(O \otimes I)|\mathrm{EPR}\rangle=\left(I \otimes O^{T}\right)|\mathrm{EPR}\rangle
$$

or graphically

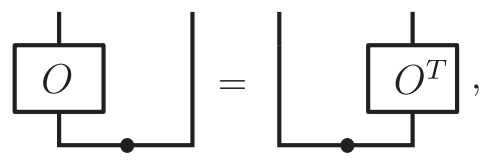

where $O^{T}$ represents the transpose of $O$. Namely, we will find the following expression:

$$
\left(I_{\bar{A}} \otimes K_{\bar{B}}\right) O(t)_{\overline{A B}}^{T}\left(I_{\bar{A}} \otimes K_{\bar{B}}^{\dagger}\right) .
$$

It is worth noting that this construction is state dependent as it depends on the initial state $(I \otimes K)|\mathrm{EPR}\rangle_{B \bar{B}}$ through its dependence on $K$. Such state-dependent constructions have been discussed in a number of previous works; see [11] for instance. operators $\Omega\left(O_{D}\right)$, supported on $C \overline{A B}$, are defined to be those which satisfy

$$
\left(O_{D} \otimes I_{C \overline{A B}}\right)|\Psi(t)\rangle \approx\left(I_{D} \otimes \Omega\left(O_{D}\right)_{C \overline{A B}}\right)|\Psi(t)\rangle,
$$

or graphically

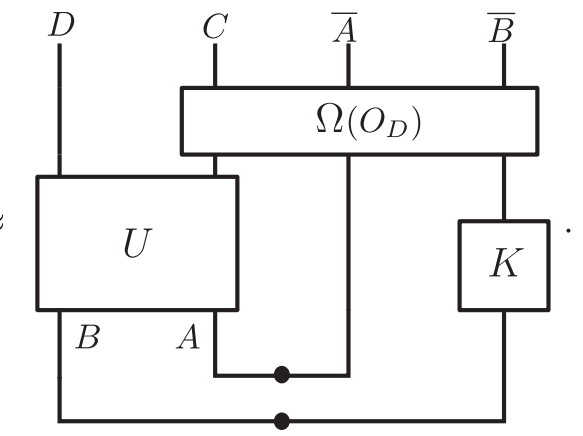

\section{B. Decoupling theorem}

It cannot be emphasized enough, however, that construction of interior partners is not unique [1]. ${ }^{4}$ Here we hope to find another expression of an interior partner operator which is independent of the initial state $(I \otimes$ $K)|\mathrm{EPR}\rangle_{B \bar{B}}$ of the black hole.

The following (informally stated) decoupling theorem, proven in a different context $[17,18]$, will be useful. A more precise version is presented in Appendix B.

Theorem 1: (Informal) Suppose that the system is scrambling in a sense of Eq. (A2) and $d_{A} \gtrsim d_{D}$. Then, the subsystems $D$ and $\bar{B}$ are decoupled (not entangled),

$$
\rho_{D \bar{B}} \approx \rho_{D} \otimes \rho_{\bar{B}}
$$

where the error is $O\left(\frac{d_{D}^{2}}{d_{A}^{2}}\right)$. Furthermore, for an arbitrary operator $O_{D}$ on the outgoing mode $D$, a partner operator $\Omega\left(O_{D}\right)_{C \bar{A}}$ can be constructed on $C \bar{A}$ without using any degrees of freedom from the early radiation $\bar{B}$.

We present a brief review of the definition of scrambling in Appendix A. Loosely speaking, it says that OTOCs decay to small values. This is a universal property of quantum black holes whose background geometries have a well-defined event horizon [16].

\footnotetext{
${ }^{4}$ One may interpret the interior partner operators as logical operators of a quantum error-correcting code where quantum information on $D$ is encoded into $C \overline{A B}$. It is then a well-known fact that logical operators have multiple equivalent expressions when their actions are restricted to a codeword subspace. The aforementioned state-dependent expression in Eq. (7) is just one possible expression of an interior operator among many others. See Sec. V for further discussions.
} 
The theorem suggests a rather surprising fact; an infalling observer's attempt to see the black hole interior will always disentangle the outgoing mode $D$ from the early radiation $\bar{B}$ due to the scrambling dynamics of the black hole. Note that, in the absence of $A$, the outgoing mode $D$ would be entangled with the early radiation $\bar{B}$, and hence the partner operator would be state dependent. Indeed, a large body of previous works $([9,11]$ for instance), without considering the effect of including an infalling observer $A$, have reached to a naive conclusion

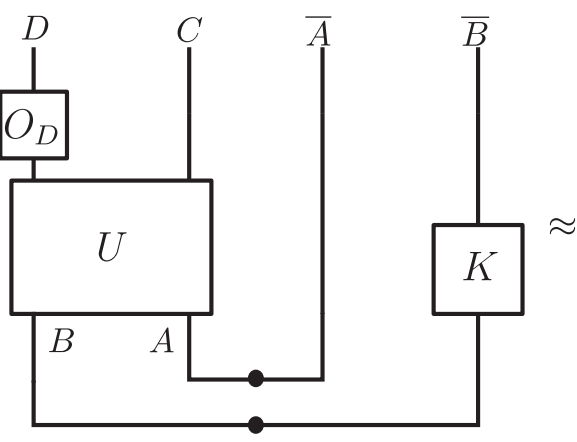

Such construction $\Omega\left(O_{D}\right)$ is state independent as it does not depend on the unitary $K$. In the next section, we will construct the interior operator $\Omega\left(O_{D}\right)$ explicitly. Namely, we will find that the construction is state independent, but is observer dependent.

It is worth emphasizing again that the disentangling phenomena occur universally due to gravitational backreaction, no matter how the infalling observer $A$ jumps into a black hole. As long as the infalling object carries a small but finite positive energy (including its rest mass), it eventually introduces significant gravitational backreaction, disentangles the outgoing radiation $D$ from the early radiation $\bar{B}$, and enables the state-independent interior operator construction. Our conclusion relies only on a widely accepted hypothesis that a black hole scrambles quantum information unitarily.

This is of course a counterargument against the so-called $\mathrm{ER}=\mathrm{EPR}$ approach [9] to the firewall puzzle by Maldacena and Susskind. Their central idea builds on the presence of preexisting entanglement (or a wormhole) between the outgoing mode $D$ and the early radiation $\bar{B}$, which is negated explicitly by the decoupling theorem. We will return to this issue in Sec. VII.

\section{STATE-INDEPENDENT INTERIOR OPERATOR}

In this section, we present an explicit construction of interior partner operators. The construction is supported exclusively on the remaining black hole $C$ and the reference system $\bar{A}$ of an infalling observer, and hence is state

\footnotetext{
${ }^{5} \mathrm{~A}$ few infalling quanta is enough to invalidate this belief.
}

that interior partner operators must be state dependent. This folklore knowledge is no longer valid once the perturbation from an infalling observer $A$ is taken into consideration. ${ }^{5}$

The theorem also suggests that, due to the monogamy of quantum entanglement, the outgoing mode $D$ is (almost) maximally entangled with the remaining black hole $C$ and the reference $\bar{A}$, which implies the existence of a partner operator supported on $C \bar{A}$. Here it is useful to represent the partner operator from theorem 1 graphically as follows:

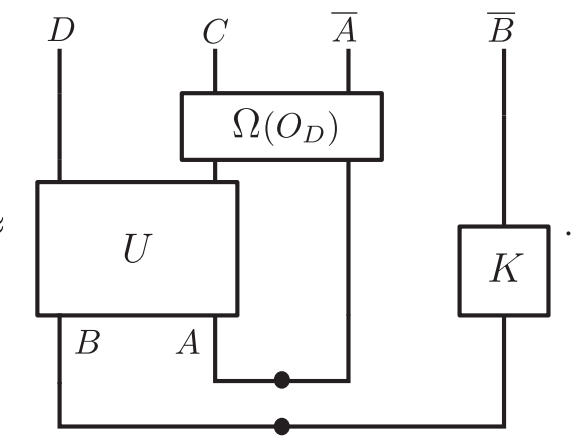

independent. In fact, the construction works not only for an old (maximally entangled) black hole, but also for a young (pure state) black hole since the expression does not involve any degrees of freedom from the early radiation $\bar{B}$ at all.

Our construction of interior operators utilizes a certain quantum operation which distills EPR pairs between $D$ and $C \bar{A}$ as this enables us to identify which degrees of freedom $D$ is entangled with. The entanglement distillation procedure is inspired by a probabilistic recovery protocol for the Hayden-Preskill thought experiment [18]. The key observation is that the interior reconstruction problem can be interpreted as the information retrieval (the Hayden-Preskill or the information loss) problem running backward in time [7]. ${ }^{6}$ This is the reason why recovery protocols for the Hayden-Preskill thought experiment can be utilized in reconstructing interior partner operators. ${ }^{7}$ A deterministic version of the recovery protocol from [18] works equally well for this purpose, but we will focus on the probabilistic one for simplicity.

\footnotetext{
${ }^{6}$ Imagine that we reverse the flow of the time so that the outgoing mode $D$ "returns" back to the black hole at time $t$ and the system evolves backward to $t=0$. Then, constructing the partner mode of $D$ is equivalent to recovering the quantum information on $D$ that has been thrown into a black hole in the reverse time flow.

${ }^{7}$ It is worth emphasizing that the interior reconstruction and the Hayden-Preskill thought experiment are different problems. Namely, the interior reconstruction works for both old and young black holes, whereas the Hayden-Preskill recovery works only for old black holes. A common mistake, frequently found in the literature, is to view the information loss problem and the firewall problem as the same thing. They are related by time reversal, but are different problems.
} 


\section{A. Entanglement distillation}

The outgoing mode $D$ is (nearly maximally) entangled with $C \bar{A}$, so it should be possible to distill EPR pairs between $D$ and $C \bar{A}$ by applying some quantum operation on $C \bar{A}$. The goal of this subsection is to present the entanglement distillation procedure.

Given the time-evolved state $|\Psi(t)\rangle$ in Eq. (2), let us construct the following state:

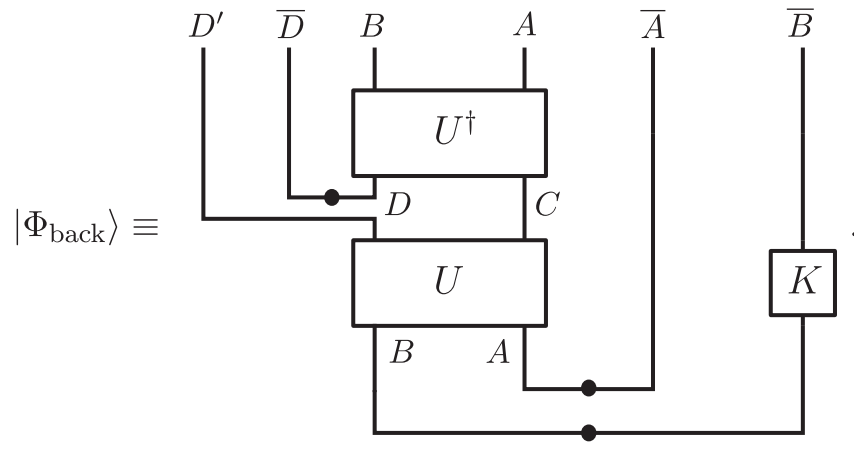

Here ancillary subsystems $D^{\prime} \bar{D}$ are introduced, qubits on $D$ are moved to $D^{\prime},|\mathrm{EPR}\rangle_{\bar{D} D}$ is prepared on $\bar{D} D$, and the timereversal $U^{\dagger}$ is applied on $D C$. Some readers may find the time-reversal $U^{\dagger}$ unphysical in a real world, but this is just a mathematical trick to obtain the interior construction.

So far, all the operations are deterministic. We now apply a projection onto EPR pairs on $A \bar{A}$. The unnormalized outcome is

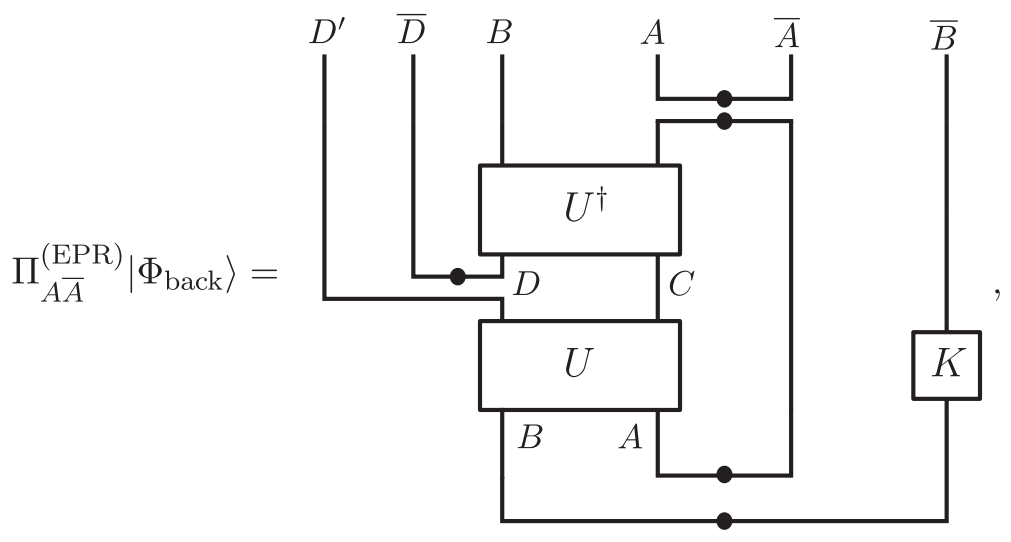

where the projector is denoted by $\Pi_{A \bar{A}}^{(\mathrm{EPR})}$. The probability amplitude for this event is

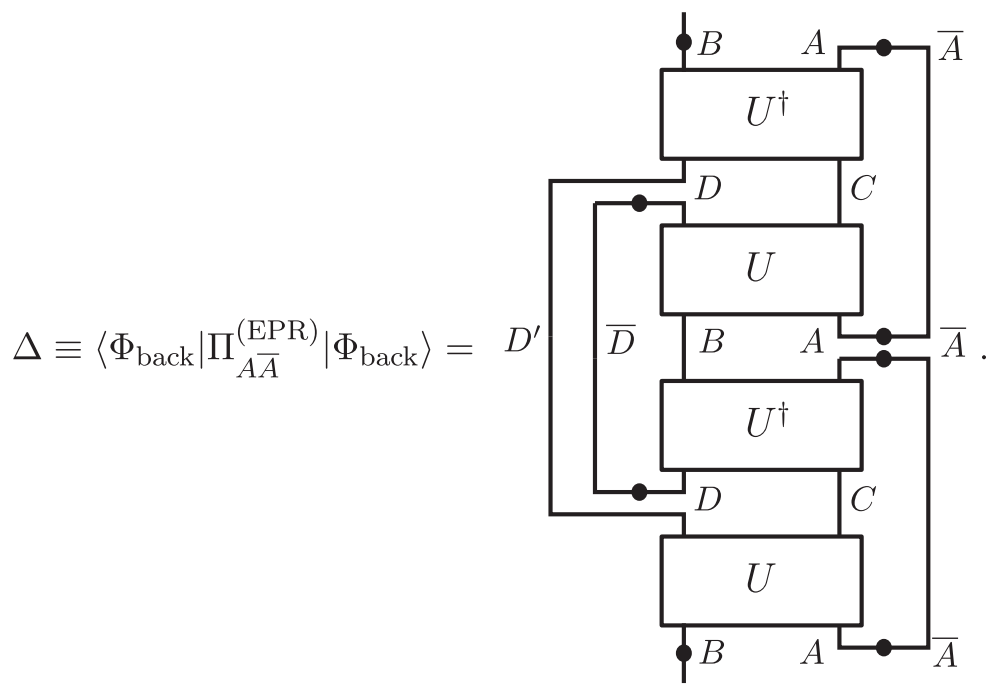


A line connecting two $B$ 's on the top and bottom of the diagram is omitted for ease of drawing. Finally, the normalized outcome after the postselection is given by

$$
\left|\tilde{\Phi}_{\text {back }}\right\rangle \equiv \frac{1}{\sqrt{\Delta}} \Pi_{A \bar{A}}^{(\mathrm{EPR})}\left|\Phi_{\text {back }}\right\rangle .
$$

Some readers may have already noticed a relation between $\Delta$ and OTOCs by staring at the diagrammatic representation of $\Delta$ where $U$ 's and $U^{\dagger}$ 's appear twice. The relation between OTOCs and $\Delta$ can be explicitly seen by the following identity [17]:

$$
\Delta=\iint\left\langle O_{A}(0) O_{D}(t) O_{A}^{\dagger}(0) O_{D}^{\dagger}(t)\right\rangle d O_{A} d O_{D},
$$

where the integrals over $O_{A}$ and $O_{D}$ represent uniform averaging over all the basis operators on $A$ and $D$, respectively. Hence, the amplitude $\Delta$ can be evaluated by using the asymptotic form of OTOCs for scrambling systems in Eq. (A2) in Appendix A. After some calculation, we obtain

$$
\Delta=\frac{1}{d_{D}^{2}}\left[1+O\left(\frac{d_{D}^{2}}{d_{A}^{2}}\right)\right] \quad\left(d_{A} \gtrsim d_{D}\right) .
$$

We now show that EPR pairs have been already distilled on $D^{\prime} \bar{D}$ after the postselection by $\Pi_{A \bar{A}}^{(\mathrm{EPR})}$. The probability of observing EPR pairs on $D^{\prime} \bar{D}$ is given by

$$
\begin{aligned}
\left\langle\tilde{\Phi}_{\text {back }}\left|\Pi_{D^{\prime} \bar{D}}^{(\mathrm{EPR})}\right| \tilde{\Phi}_{\text {back }}\right\rangle & =\frac{1}{\Delta}\left\langle\Phi_{\text {back }}\left|\Pi_{A \bar{A}}^{(\mathrm{EPR})} \Pi_{D^{\prime} \bar{D}}^{(\mathrm{EPR})}\right| \Phi_{\text {back }}\right\rangle \\
& =\frac{1}{\Delta} \frac{1}{d_{D}^{2}} \\
& \approx 1 .
\end{aligned}
$$

In the second step, we have used the following identity:

\section{B. Construction of interior operator}

To construct interior partner operators $\Omega\left(O_{D}\right)$ for the perturbed black hole state $|\Psi(t)\rangle$, we need to "undo" the projection $\Pi_{A \bar{A}}$ and the time-reversal $U^{\dagger}$. Partner operators are explicitly given by

$$
\begin{aligned}
\Omega\left(O_{D}\right) \equiv & \frac{1}{\Delta} \frac{1}{d_{D}} \operatorname{Tr}\left[\left(O_{D} \otimes I_{C \bar{A}}\right)\left(U_{B A} \otimes I_{\bar{A}}\right)\right. \\
& \left.\times\left(I_{B} \otimes \Pi_{A \bar{A}}^{(\mathrm{EPR})}\right)\left(U_{B A}^{\dagger} \otimes I_{\bar{A}}\right)\right],
\end{aligned}
$$

or graphically
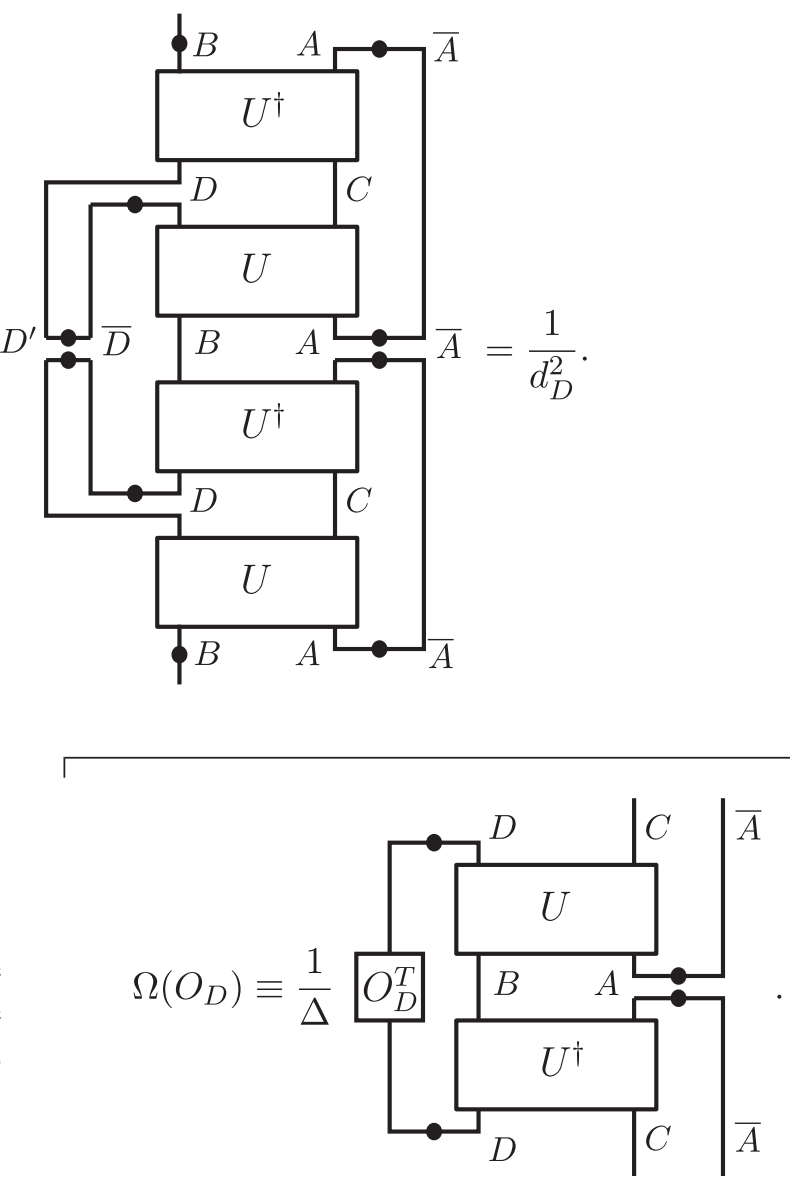

Most readers will not find this expression particularly illuminating. In the next section, we will further simplify the expression and discuss its relation to the boundary operator's growth. 
It can be verified that these are appropriate partner operators by evaluating the overlap between $\left(O_{D} \otimes I\right)|\Psi(t)\rangle$ and $\left(I \otimes \Omega\left(O_{D}\right)\right)|\Psi(t)\rangle$,

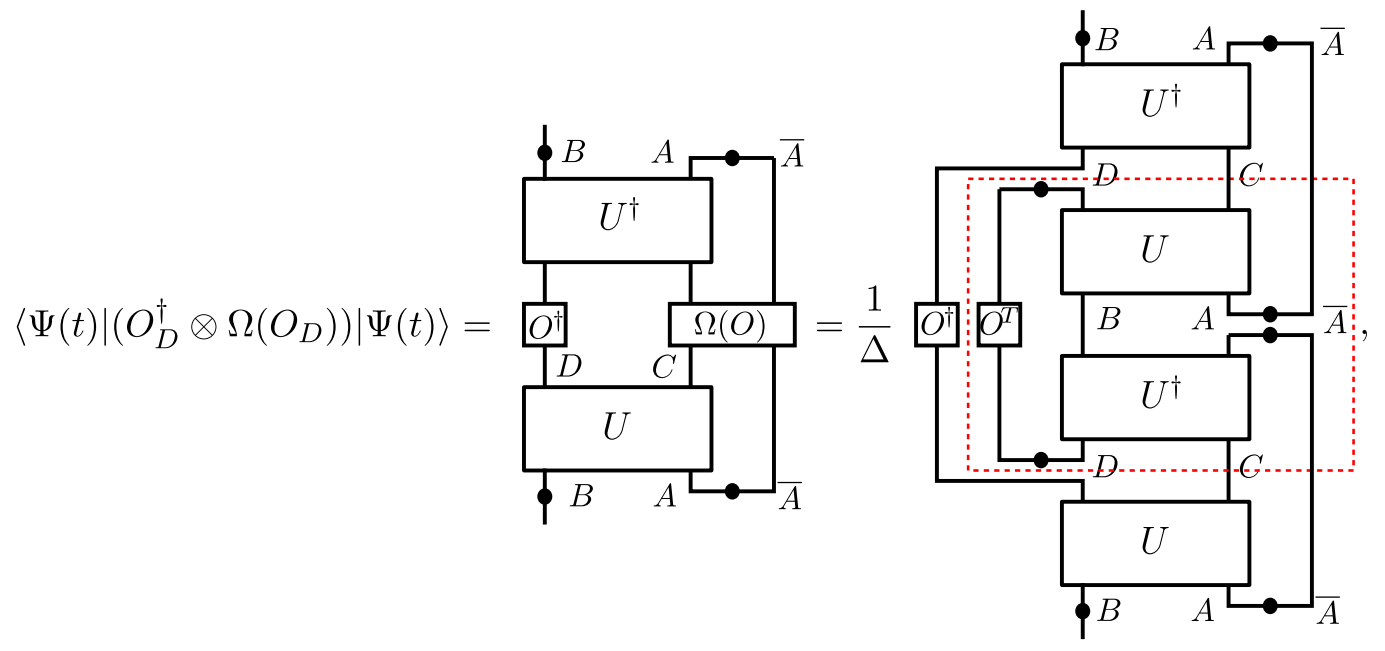

where a part enclosed by a dotted square corresponds to $\Omega\left(O_{D}\right)$. Taking average over basis operators on $D$, we have

$$
\begin{aligned}
& \int d O_{D}\left\langle\Psi(t)\left|\left(O_{D}^{\dagger} \otimes \Omega\left(O_{D}\right)\right)\right| \Psi(t)\right\rangle \\
& =\frac{1}{\Delta}\left\langle\Phi_{\text {back }}\left|\Pi_{A \bar{A}}^{(\mathrm{EPR})} \Pi_{D^{\prime} \bar{D}}^{(\mathrm{EPR})}\right| \Phi_{\text {back }}\right\rangle=\frac{1}{\Delta} \frac{1}{d_{D}^{2}} \approx 1,
\end{aligned}
$$

where we used $\int d O O \otimes O^{*}=|\mathrm{EPR}\rangle\langle\mathrm{EPR}|$. Hence, $\left\langle\Psi(t)\left|\left(O_{D}^{\dagger} \otimes \Omega\left(O_{D}\right)\right)\right| \Psi(t)\right\rangle \approx 1$ for each $O_{D}$. It is worth emphasizing that our construction has a concrete operational meaning: It is constructed by distilling EPR pairs between the outgoing mode $D$ and the remaining black hole $C$ plus the reference $\bar{A}$. Indeed, the average overlap in Eq. (21) is equal to the EPR distillation probability in Eq. (16).

\section{Pure state observer}

Some readers may find the use of a reference system $\bar{A}$ a bit uncomfortable. Instead of introducing a reference system $\bar{A}$ entangled with $A$, let us consider a scenario where a measurement probe $A$, prepared in a particular pure state (e.g., $|0\rangle$ ), is dropped toward the black hole.

The procedure to distill EPR pairs and construct interior operators proceeds in an analogous manner except that this time we need to project $A$ onto $|0\rangle$,

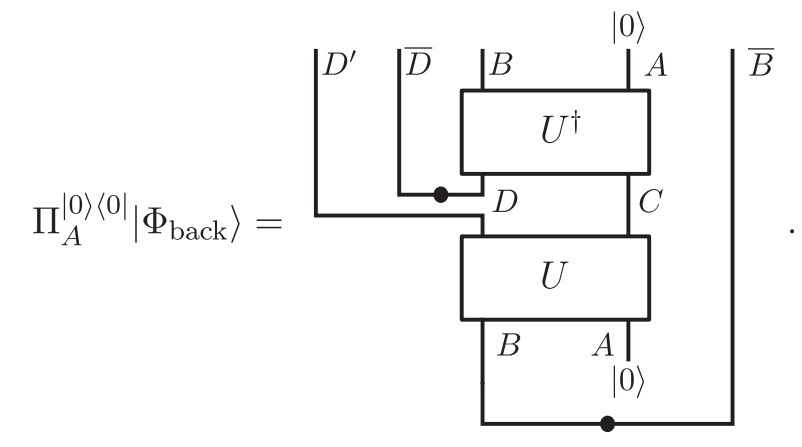

The probability amplitude for the projection onto $|0\rangle$ can be computed from the OTOC asymptotics,

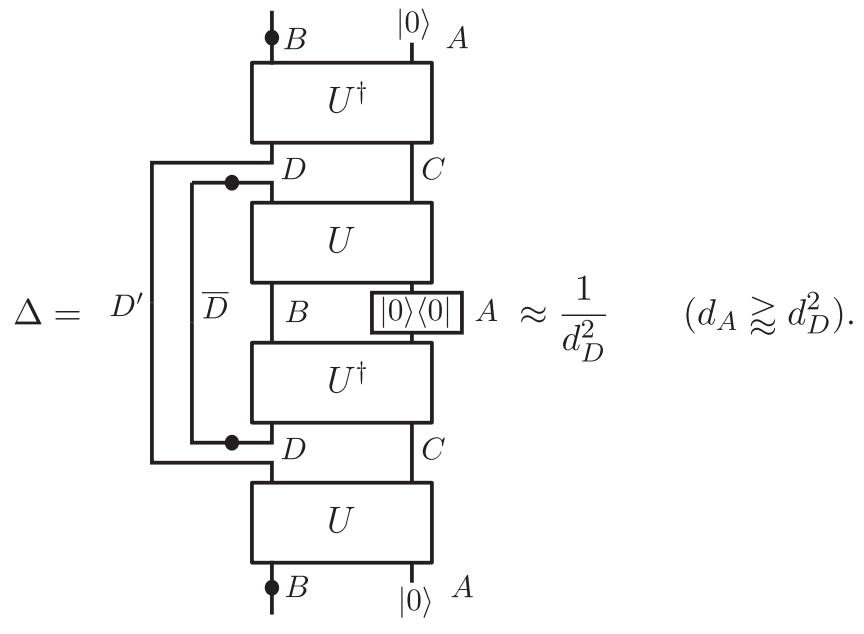


Note that the reconstruction criteria become $d_{A} \gtrsim d_{D}^{2}$ instead of $d_{A} \gtrsim d_{D}$. In the qubit count, $A$ needs to contain more than twice qubits on $D$.

The probability of observing EPR pairs on $D^{\prime} \bar{D}$ is $\frac{1}{\Delta}\left\langle\Phi_{\text {back }}\left|\Pi_{A}^{|0\rangle\langle 0|} \Pi_{D^{\prime} D}\right| \Phi_{\text {back }}\right\rangle=\frac{1}{\Delta} \frac{1}{\Delta d_{D}^{2}} \approx 1$. Interior partner operators are given by

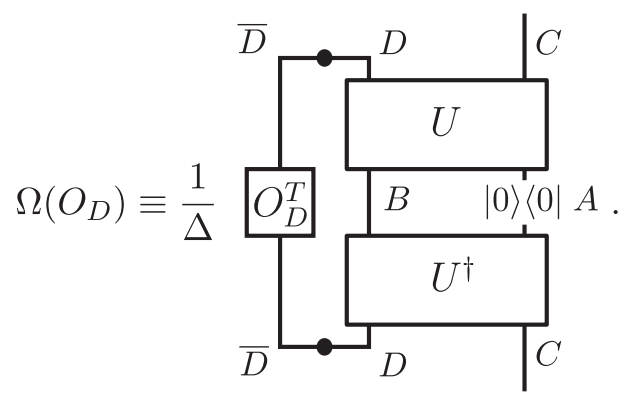

\section{Young black hole interior}

So far, we have focused on an old black hole that is maximally entangled with the early radiation $\bar{B}$. Rather surprisingly (but in some sense quite naturally), our construction remains valid for a young black hole which has not yet emitted the Hawking radiation and can be represented as a pure state.

This is simply because the expression of interior operator does not involve the early radiation $\bar{B}$ at all. To be explicit, let us consider the initial state of the following form:

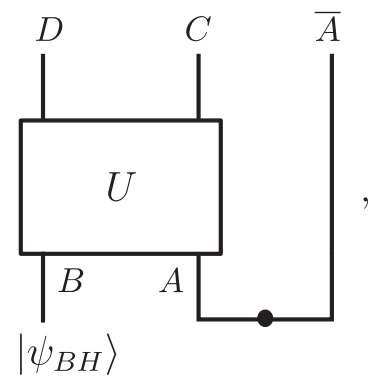

where $\left|\psi_{\mathrm{BH}}\right\rangle$ represents the pure state of a young black hole on $B$. Then, in evaluating Eq. (20), we simply insert $\left|\psi_{\mathrm{BH}}\right\rangle\left\langle\psi_{\mathrm{BH}}\right|$, instead of the maximally mixed state $\frac{1}{d_{B}} \mathbb{I}_{B}$, to the line corresponding to $B$. This replacement does not affect the outcome of Eq. (21), showing that the same construction $\Omega\left(O_{D}\right)$ works as an interior operator for a young black hole as well. Hence, we conclude that our construction of interior partner operator is state independent and works for both young and old black holes.

Our construction provides a possible resolution to a version of the firewall puzzle for black hole microstates due to Marolf and Polchinski [19]. They argued that, if an interior operator is state independent, then there must exist a firewall for typical black hole microstates. Much of previous works, including the ER $=\mathrm{EPR}$ approach by Maldacena and Susskind, then speculated that interior operators must be state dependent in order to avoid the firewall. Here we have avoided the no-go result by Marolf and Polchinski by considering the effect of an infalling observer and explicitly demonstrated that state-independent interior operators can be written down. It is again worth emphasizing that our conclusion relies only on a widely accepted hypothesis that a black hole scrambles quantum information unitarily.

In later sections, we will see that the possibility of stateindependent interior construction not only reveals fundamental flaws in the ER $=E P R$ approach to the firewall puzzle, but also suggests a rather different scenario for resolving the firewall puzzle. ${ }^{8}$

\section{E. Seeing the interior}

We conclude this section by presenting a possible bulk quantum gravity interpretation of the entanglement distillation. Imagine that the entanglement distillation protocol is actually implemented on the boundary quantum mechanical system. The fact that an EPR pair is distilled between $\tilde{D}$ and the outgoing mode $D$ suggests that the partner mode $\tilde{D}$ has been extracted as some simple degrees of freedom on the boundary. A possible physical interpretation is that the interior partner mode $\tilde{D}$ is brought to the AdS boundary from the black hole interior by the entanglement distillation protocol. Also, the fact that the EPR entanglement between $A$ and $\bar{A}$ has been restored via the EPR projection $\Pi_{A \bar{A}}^{(\mathrm{EPR})}$ suggests that the infalling observer $A$ has returned to the exterior from her journey to the black hole interior. As such, the infalling observer $A$, who crossed the black hole horizon, may return to the black hole exterior with the interior partner mode $\tilde{D}$ in her hand by implementing the entanglement distillation.

\section{OPERATOR GROWTH INTERPRETATION}

In this section, we will relate the aforementioned construction to the operator growth interpretation of scrambling [14]. The operator growth picture relies crucially on the finiteness of the Hilbert space. It is an interesting future problem to generalize our construction to quantum field theories. See [21] for discussions on oscillator systems.

\section{A. Truncation by local operator}

Scrambling phenomena can be associated with the boundary operator's growth. Given a single qubit operator

\footnotetext{
${ }^{8}$ There are two versions of the firewall puzzle: (i) for an old black hole and (ii) for generic black hole pure states [19,20]. It is rather strange to try to resolve the puzzle by treating black holes with preexisting entanglement [the case (i)] in a special manner as in the ER = EPR approach. Our approach resolves the puzzle for both cases on an equal footing as we will see in Sec. VI.
} 
$V$ in a system of $n$ qubits, consider its time evolution by the Hamiltonian $V(t)=e^{-i H t} V e^{i H t}$,

$$
\begin{aligned}
V(t)= & V-i H t[H, V]-\frac{t^{2}}{2 !}[H,[H, V]] \\
& +\frac{i t^{3}}{3 !}[H,[H,[H, V]]]+\cdots
\end{aligned}
$$

Commutators $[H, \cdots[H,[H, V]]]$ generically increase the number of supports and $V(t)$ becomes a high weight operator for large $t$. The growth of $V(t)$ can be probed by OTOCs. When $W$ is some other single qubit operator on a distant qubit and $V(t)$ has nontrivial support on that qubit, $\langle V(t) W(0) V(t) W(0)\rangle$ decays from its initial value.

The operator growth can be related to interior reconstruction by considering truncation of operators. For an arbitrary operator $V$ on a Hilbert space $\mathcal{H}=\mathcal{H}_{C} \otimes \mathcal{H}_{D}$, its truncation by $W_{D}$ is defined by

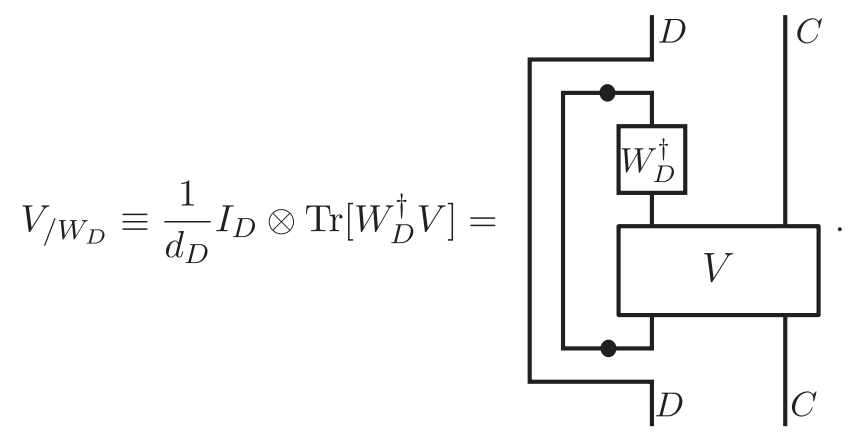

$$
\Omega\left(O_{D}\right)=\frac{1}{\Delta} \int d O_{A} O_{D}{ }_{B}^{T}
$$

The truncation $V_{/ W_{D}}$ is constructed by picking up terms with the integral over basis operators on $A$. The reason why the backward evolution $O_{A}(-t)$ appears can be understood by rewriting OTOCs as $\left\langle O_{D}^{\dagger}(t) O_{A}^{\dagger}(0) O_{D}(t) O_{A}(0)\right\rangle=$ $\left\langle O_{D}^{\dagger}(0) O_{A}^{\dagger}(-t) O_{D}(0) O_{A}(-t)\right\rangle$ which evaluates the overlap between $O_{A}(-t)$ and $O_{D}(0)$.

Some readers may feel uncomfortable about taking an average over $O_{A}$. It turns out that we can safely ignore averaging in most cases. When $A$ is sufficiently large, we may pick one Pauli operator $O_{A}$ at random to construct$$
\prod_{\bar{A}}^{\bar{A}}=\frac{1}{\Delta} \int d O_{A} O_{A}^{\dagger}(-t)_{O_{D}^{\dagger}} \otimes O_{\bar{A}}^{T}
$$

Note that truncated operator $V_{/ W_{D}}$ acts nontrivially only on $C$. In the Choi representation $|O\rangle \equiv(O \otimes I)|\mathrm{EPR}\rangle$, the truncation can be defined by $\left|V_{/ W_{D}}\right\rangle \equiv\left|I_{D}\right\rangle\left\langle W_{D} \mid V\right\rangle$.

To gain some intuition, let us expand $V$ in terms of Pauli operators: $V=\sum_{P \in \text { Pauli }} \alpha_{P} P$. Assume that $D$ consists of just a single qubit and choose $W_{D}$ to be a Pauli operator $\left\{I_{D}, X_{D}, Y_{D}, Z_{D}\right\}$. Define four sets of Pauli operators on $\mathcal{H}$, depending on the Pauli operator supported on $D$,

$$
\begin{aligned}
\text { Pauli }\left.\right|_{P_{D}} & =\left\{P_{D} \otimes \text { Pauli Operators on } C\right\} \\
P_{D} & =I_{D}, X_{D}, Y_{D}, Z_{D} .
\end{aligned}
$$
with Pauli operators containing $W_{D}$ on $D$,

$$
V_{/ W_{D}}=\sum_{P \in \text { Pauli }_{W_{D}}} \alpha_{P} P
$$

In other words, the truncation gives rise to nontrivial operators when the original operator $V$ and the target operator $W_{D}$ "collide" with each other.

\section{B. Interior operator from collision}

The notion of truncation simplifies the expression of interior operators. Using

$$
\int d O_{A} O_{A} \otimes O_{\bar{A}}^{*}=|\mathrm{EPR}\rangle\left\langle\left.\mathrm{EPR}\right|_{A \bar{A}},\right.
$$

the interior partner operator in Eq. (19) can be expressed concisely as

a partner operator $\frac{1}{\Delta} O_{A}^{\dagger}(-t)_{/ O_{D}^{\dagger}} \otimes O_{\bar{A}}^{T}$ without taking averages since statistical variances are suppressed in a scrambling system. Then the relation between the operator growth and interior operator construction becomes transparent; when $O_{A}(-t)$ collides with the target outgoing operator $O_{D}$, interior partner operators can be constructed.

For the cases with a pure state input $|0\rangle$ on $A$, we obtain an even simpler expression 


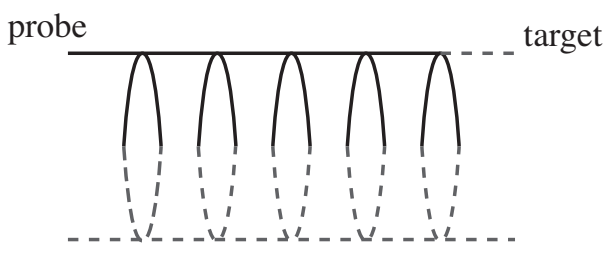

FIG. 3. The partner operator as the upper half of the ladder diagram $(q=4 \mathrm{SYK})$.

$$
\Omega\left(O_{D}\right)=\frac{1}{\Delta} O_{A}(-t)_{/ O_{D}^{\dagger}} \quad O_{A}=I_{B} \otimes|0\rangle\left\langle\left. 0\right|_{A} .\right.
$$

The physical interpretation is clearer in this case. Recall that $|0\rangle$ was the initial state of the infalling observer and $O_{A}$ is its projection. Hence, the interior partner operators are constructed due to the gravitational collision between the infalling observer and the target outgoing $O_{D}$. This suggests that the interior modes are created once the outgoing modes are measured by the infalling observer.

\section{On SYK model}

Finally, we make a brief comment on interior operators for the SYK model. Since the SYK model exhibits scrambling dynamics, our prescription can be applied to obtain the entangled partner. It has a simple Feynman diagrammatic interpretation modulo one technical subtlety.

Consider the OTOC $\left\langle\psi_{1}(0) \psi_{N}(t) \psi_{1}(0) \psi_{N}(t)\right\rangle$ where $\psi_{1}, \psi_{N}$ are interpreted as infalling and outgoing modes, respectively. Here we would like to construct a partner of $\psi_{N}$. The dominant contribution to OTOCs comes from the ladder diagram; see Fig. 3. In [22], it has been pointed out that the ladder diagram characterizes the collision between $\psi_{1}(-t)$ and $\psi_{N}(0)$. In this interpretation, the upper half of the ladder diagram can be viewed as the dominant contribution in the growth of $\psi_{1}(-t)$. Hence, by truncating $\psi_{N}$ from the upper half of the ladder diagram, we obtain the interior operator (Fig. 3).

It should be however noted that the above diagrammatic interpretation has a problem. The ladder diagram accounts for the dominant piece in the OTOC decay until the scrambling time. Around the scrambling time, the subleading terms become important. Since the fidelity of our reconstruction becomes better when OTOCs have decayed to small values, the diagrammatic expression in Fig. 3 is a good description of interior partner operator only for some time window.

\section{OBSERVER-DEPENDENT INTERIOR}

We have seen that the black hole interior operator can be constructed in a way independent of the black hole's initial state due to the infalling observer's backreaction. Yet, it is important to recognize that the construction is dependent on how the infalling observer $A$ is introduced to the system. Hence the construction is state independent but is observer dependent. This suggests that the black hole interior should be considered as observer dependent. ${ }^{9}$ In fact, we have already seen observer-dependent nature of the black hole interior rather vividly in the operator growth interpretation. We found that the interior operator can be constructed by examining the collision between the outgoing mode $W$ and the infalling mode $V(-t)$. The implication is that the interior mode was created dynamically once the infalling observer measures the outgoing mode. This interpretation further hints observer dependence of the black hole interior.

We may formalize the notion of observer dependence by borrowing a quantum information theoretic language. Let us consider a maximally entangled black hole and recall how the interior modes are realized for the outside and infalling observers, respectively. In the absence of the infalling observer, the entangled partner of the outgoing mode can be found as some degrees of freedom in the early radiation. This is what the outside observer perceives as the interior mode. Yet, from the perspective of the infalling observer, this thing in the early radiation is not the interior mode anymore since it has been already decoupled from the outgoing mode. The infalling observer will perceive the mode which we have identified in previous sections as the true interior partner mode.

As is evident from this argument, observer dependence of the black hole interior is closely related to the fact that expressions of interior partner operators are not unique. This property can be better understood by using an analogy with quantum error-correcting codes. In the language of quantum error-correcting code, partner operators can be interpreted as logical operators where quantum information on the outgoing mode $D$ is encoded into $C \overline{A B}$. Consider the following embedding map $\Gamma: \mathcal{H}_{D} \rightarrow \mathcal{H}_{\text {code }}$ defined by

$$
\begin{aligned}
|\psi\rangle & \in \mathcal{H}_{D} \stackrel{\Gamma}{\rightarrow} \Gamma(|\psi\rangle) \\
& =\left\langle\left.\psi\right|_{D} \mid \Psi(t)\right\rangle_{D C \overline{A B}} \in \mathcal{H}_{\text {code }} \subseteq \mathcal{H}_{C \overline{A B}},
\end{aligned}
$$

where we omitted the normalization factor for simplicity. For readers familiar with tensor diagrammatic notations, it is convenient to represent the embedding map graphically as follows by rotating the original diagram for $|\Psi(t)\rangle$ 180 degree:

\footnotetext{
${ }^{9}$ Observer dependence of a black hole itself might sound like an old and standard idea from the perspective of its thermodynamic property. For instance, the no hair theorem tells that black holes in thermal equilibrium are characterized by a few macroscopic observables. This suggests that whatever happened in the past will not affect the present observer as far as outgoing modes are concerned. A typical time scale for this is order of the thermal time. What we advocate here is distinct from this conventional wisdom based on the thermodynamics of black holes. We argue that the black hole interior, which is understood as entangled partners of outgoing modes, must be observer dependent too. A typical time scale for this is order of the scrambling time.
} 


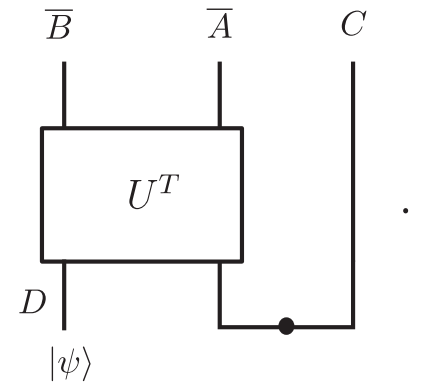

Here partner logical operators are those which may transform wave functions in the code subspace $\mathcal{H}_{\text {code }}$ in a nontrivially manner. In the quantum error-correction interpretation, logical operators on $\overline{A B}$ is what the outside observer perceives as the entangled partner whereas those on $C \bar{A}$ is what the infalling observer will measure when approaching the horizon.

We have argued that quantum error-correcting nature of interior reconstruction gives rise to observer-dependence. At this point, it is interesting to recall that the holographic mapping from bulk operators to boundary operators in the AdS/CFT correspondence can be understood as a quantum error-correcting code. The canonical example of holographic quantum error correction is the empty AdS space where the bulk operator at the center can be reconstructed on any interval which is longer than the half of the system via the AdS/Rindler reconstruction. Our interpretation is that each boundary expression corresponds to what the boundary observer, who has an access to the given boundary interval, perceives as the bulk operator.

We conclude this section by recalling a certain argument by Hayden and Preskill [15]. Let us consider two observers who jump into the one-sided black hole and try to meet inside the black hole. If the time separation $\Delta t$ between them is longer than the scrambling time, an observer needs large amount of energy, larger than the Planck energy, to send a signal to the other observer. Hence, two observers will not be able to meet. This was the crucial observation by Hayden and Preskill which led to the lower bound on the scrambling time. Our interpretation of their argument is as follows. When two observers are separated by more than the scrambling time, the black hole interiors they observe are unrelated. They can enjoy their own interiors without being perturbed by others. See [23] for further discussions.

\section{ON BLACK HOLE PUZZLES}

In this section, we shall discuss implications of state independence and observer dependence on several important conceptual puzzles concerning the black hole interior, such as the firewall puzzle [12,20,24,25] and the MarolfWall puzzle [26]. Namely, we will propose novel resolutions of these puzzles by relying on the lessons we have learned from the interior reconstruction. We also make some comments on the relevance (and the irrelevance) between the firewall problem and the information loss problem since two problems are often (wrongly) treated as the same issue in the community.

\section{A. Firewall puzzle}

Let us briefly recall the firewall argument $[12,24,25]$ (Fig. 4). Given an old black hole $B$ which is maximally entangled with the early radiation $R$, let us consider an outgoing mode $D$ which was just emitted from the black hole. Since the black hole is maximally entangled, the outgoing mode $D$ must be entangled with some degrees of freedom in $R$. Then the outside observer, Bob, may distill a qubit $R_{D}$ from $R$ that is entangled with the outgoing mode $D$ and hand it to the infalling observer Alice who is going to jump into a black hole. After crossing the smooth horizon, Alice will see an interior mode $\tilde{D}$ which is entangled with the outgoing mode $D$. This, however, leads to a contradiction because $D$ is also entangled with $R_{D}$. If we believe what Bob says, $D$ has to be entangled with $R_{D}$, so $D$ and $\tilde{D}$ are not entangled, leading to possible existence of some high energy density at the horizon.

The aforementioned reconstruction of interior partner operators naturally guides us to a resolution of the firewall puzzle [8]. The resolution is immediate from a physical interpretation of the aforementioned reconstruction of the interior partner operators. When Alice falls into a black hole, the outgoing mode $D$ is disentangled from the distilled qubit $R_{D}$ due to the backreaction which makes OTOCs decay. In fact, the decoupling theorem 1 tells that $D$ is decoupled from any degrees of freedom in the early radiation $R$. Hence, the monogamy of quantum entanglement is not violated. Alice will be able to observe the interior mode $\tilde{D}$ which is distinct from the original partner mode $R_{D}$.

It is worth discussing how this proposal resolves the puzzle in the form of the AMPS thought experiment. In the AMPS thought experiment, the outside observer hands distilled qubits $R_{D}$ to the infalling observer who attempts to verify the violation of the monogamy of entanglement. So,

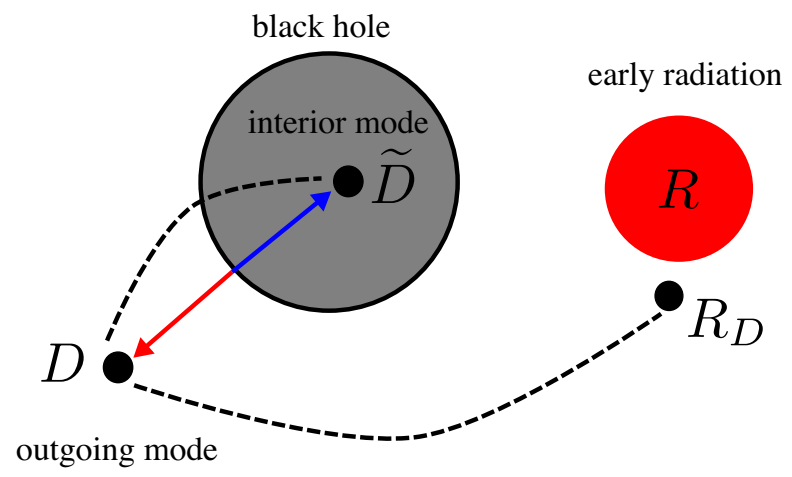

FIG. 4. Summary of the firewall argument. The outgoing mode $D$ cannot be entangled with the early radiation $R$ and the interior mode $\tilde{D}$ simultaneously. 
(a)

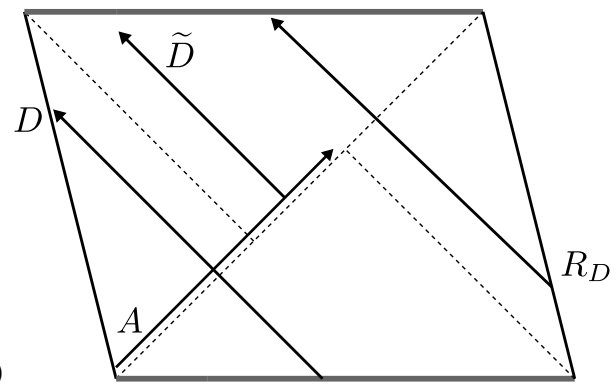

(b)

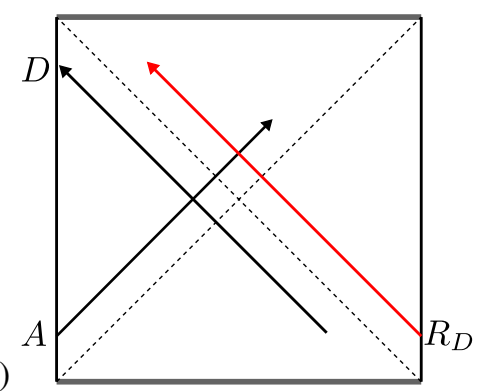

FIG. 5. (a) The bulk interpretation of the proposed resolution of the firewall puzzle. The infalling observer $A$ is treated as a gravitational shockwave. (b) A schematic picture of previous proposals. This is not consistent with unitarity or scrambling of the black hole dynamics.

the infalling observer should carry more qubits than $R_{D}$ when she crosses the black hole horizon. This effectively creates a situation with $d_{A} \geq d_{D}$. Then, from the decoupling theorem, one can easily show that $D$ is no longer entangled with $R_{D}$ due to scrambling dynamics. See Appendix $\mathrm{C}$ for more details.

The bulk interpretation of the aforementioned resolution can be obtained by treating the backreaction from Alice as a gravitational shockwave [Fig. 5(a)]. For simplicity of drawing, let us consider the AdS eternal black hole. Given the outgoing mode $D$, one possible representation of interior partner operators can be constructed by time evolving a corresponding mode on the right-hand side. This mode, constructed exclusively on the degrees of freedom on the right-hand side, was called $R_{D}$. We now include the effect of the infalling observer $A$ as a gravitational shockwave and draw the backreacted geometry where the horizon is shifted as depicted in Fig. 5(a). If the time separation between the outgoing mode $D$ and the infalling observer $A$ is longer than or equal to the scrambling time, the interior mode $\tilde{D}$, which is distinct from $R_{D}$, can be found across the horizon.

We also address a version of the firewall puzzle for typical state black holes due to Marolf and Polchinski [19]. Their argument can be summarized as follows. If $O_{\tilde{D}}$ is a partner operator of $O_{D}$, they should satisfy $\left\langle\psi_{\mathrm{BH}}\right| O_{D} \otimes$ $O_{\tilde{D}}\left|\psi_{\mathrm{BH}}\right\rangle \simeq 1$ where $\left|\psi_{\mathrm{BH}}\right\rangle$ is the black hole's pure state. Suppose that $O_{\tilde{D}}$ is a partner operator for all $\left|\psi_{\mathrm{BH}}\right\rangle$ and is independent of $\left|\psi_{\mathrm{BH}}\right\rangle$. Then we have

$$
\int d\left|\psi_{\mathrm{BH}}\right\rangle\left\langle\psi_{\mathrm{BH}}\left|O_{D} \otimes O_{\tilde{D}}\right| \psi_{\mathrm{BH}}\right\rangle=\frac{1}{d} \operatorname{Tr}\left(O_{D} \otimes O_{\tilde{D}}\right)=0
$$

for traceless $O_{D}$, leading to a contradiction. Thus, Marolf and Polchinski concluded that either (i) a partner operator $O_{\tilde{D}}$ does not exist (i.e., the firewall exists for typical $\left|\psi_{\mathrm{BH}}\right\rangle$ ), or (ii) $O_{\tilde{D}}$ depends on $\left|\psi_{\mathrm{BH}}\right\rangle$. We have avoided this puzzle due to Marolf and Polchinski by noting that a partner operator $O_{\tilde{D}}$ can be independent of $\left|\psi_{\mathrm{BH}}\right\rangle$ when an infalling observer is treated as an actual physical object with finite energy. The bulk interpretation is straightforward from a reasoning similar to Fig. 5(a) where an infalling observer $A$ creates her own interior geometry by her backreaction.

Below is a side comment. Scrambling phenomena have to do with entanglement dynamics which can be measured either by preparing two entangled copies of the same system or by evolving the system forward and backward in time. It is quite interesting that the firewall problem can be resolved in a way which crucially relies on entanglement dynamics, but does not appear to involve time reversal or two copies of the system. The key to understand this puzzle is that the infalling observer, not the outside observer, will be the one to measure quantum entanglement by seeing the smoothness of the horizon. To communicate her infalling experience back to the outside observer, she needs to be pulled back to the outside. In our construction of interior operators, this was done by performing the EPR distillation. We may use the deterministic variant from [18] if we want to save her for sure. This procedure does involve time reversal which is required for verification of entanglement from the perspective of the outside observer.

\section{B. Rendezvous in black hole?}

Observer dependence due to gravitational backreaction also provides a possible resolution of another interesting puzzle concerning the Hilbert space structure of two-sided black holes. Consider the two-sided AdS black hole geometry which is usually interpreted as the thermofield double state of the left and right CFTs whose Hilbert space has a factorized form $\mathcal{H}=\mathcal{H}_{L} \otimes \mathcal{H}_{R}$. In a naive semiclassical treatment on a fixed background geometry, it appears that two observers from the left and the right can be arranged to meet inside the black hole if they depart sufficiently before $t=0$. See Fig. 6. But the conformal field theory (CFT) Hamiltonian $H_{L}+H_{R}$ does not couple two sides, so two observers should not be able to meet. How do we make sense of these?

A possible resolution of this puzzle was suggested by Marolf and Wall [26]. This puzzle itself was referred to as the Marolf-Wall puzzle in [27]. Their proposal to resolve this puzzle was that we need additional degrees of freedom 


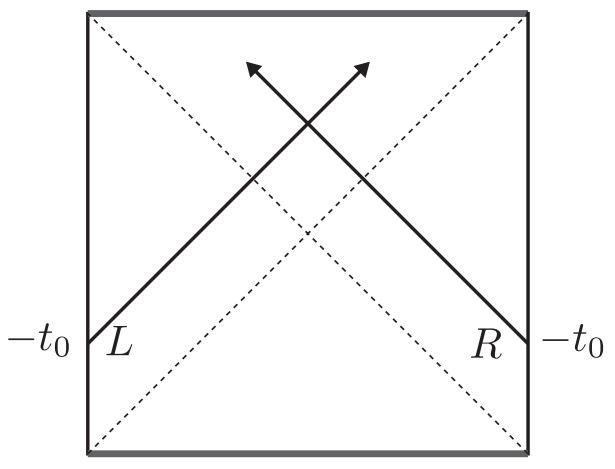

FIG. 6. Classical trajectories from the left and right sides can intersect inside the black hole. Can two observers meet?

beyond the original CFTs, some sort of superselection sectors, in order to describe the meeting. This proposal is closely related to the tensor factorization problem as pointed out in [28].

Here, however, we arrive at a different resolution of the puzzle by considering the effect of backreaction. In fact, the structure of the problem is identical to the one in the firewall puzzle. Suppose that two signals were sent at $t=-t_{0}$ so that their semiclassical trajectories would intersect in the black hole interior (Fig. 6). For definiteness, we focus on the left signal $L$ as the one we start with and ask if it receives the right signal $R$ in the interior or not. In the AdS/CFT correspondence, the right signal $R$ can be interpreted as an excitation on the right CFT. Then, in the absence of the left signal, one can find some mode $R^{\prime}$ on the left side at time $t=+t_{0}$ which is entangled with the mode $R$. In the unperturbed geometry, the right signal, started at $t=-t_{0}$, would travel along the mode $R$ which is realized as the entangled partner of $R^{\prime}$. However, due to the backreaction by the left signal $L$, the near-horizon geometry will be shifted in the same manner as in the firewall problem. From the reasoning similar to Sec. VI A, we see that the right signal will not reach the left signal if $t_{0} \gtrsim t_{\mathrm{scr}}$. The original entangled partner mode $R$, where the right signal was supposed to travel along, is no longer entangled with the left side $R^{\prime}$ due to the backreaction. Inside the black hole, the left signal $L$ will come across the newly created partner mode $\tilde{R}$ where the right signal is absent. If $t_{0} \ll t_{\text {scr }}$, two signals would meet near the singularity where we should doubt the validity of semiclassical treatment from the beginning.

The central idea behind this resolution is again that the black hole interior is observer dependent. Left and right observers jump into their own black hole interiors which are separated degrees of freedom. Given a possible relation between the Marolf-Wall puzzle and the tensor factorization problem, we speculate that observer dependence of black hole interiors may also provide useful insights on the latter problem.

\section{Page curve and state dependence}

Finally, we discuss the implication of our stateindependent reconstruction to the information loss problem. There are many variants of the black hole information puzzle, but we shall focus on the particular question of why the entanglement entropy starts to decrease after the Page time [29].

In the literature, we often see that the Page curve problem and the firewall problem are discussed on the equal footing as if these have the same origin; see $[11,30]$ for instance. While both problems are concerned about entangled partners of outgoing modes, their resolutions come from entanglement properties of completely opposite nature as we clarify below.

The initial increase of the entanglement entropy results from the fact that the outgoing mode is entangled with the remaining black hole degrees of freedom. After the Page time, however, the situation will change drastically. If unitarity is assumed from the outside observer's description, the outgoing mode after the Page time must be somehow entangled with the early radiation since otherwise the entanglement entropy will not decrease. This observation seems to suggest that the interior partner of the late outgoing mode must be constructed by using the early radiation degrees of freedom and hence must be state dependent. It appears to be in tension with our view that interior partner operators can be reconstructed in a stateindependent manner.

Our proposal to resolve this apparent tension is that in an evaporating black hole, a large portion of outgoing modes can be still reconstructed in a state-independent manner, but a tiny portion accounting for the black hole evaporation must be state dependent.

As a warm-up, let us recap the interior reconstruction problem for a large AdS black hole which does not evaporate. The time evolution of this quantum system can be depicted as follows:

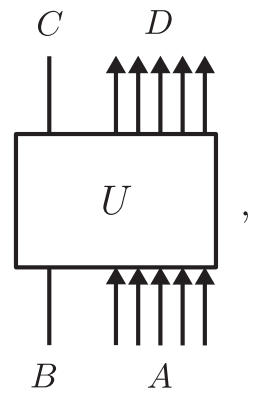

where $A, D$ can be interpreted as infalling and outgoing modes, respectively, while $B, C$ are all the other degrees of freedom. State-independent reconstruction is possible as long as $d_{A} \gtrsim d_{D}$. This condition is satisfied in the $\operatorname{AdS}$ 
black holes where the outgoing modes are reflected back at the boundary and hence $d_{A}=d_{D}{ }^{10}$

For evaporating black holes, however, there will be more outgoing modes than infalling modes, corresponding to the situation with $d_{D} \gtrsim d_{A}$. Let us examine how the stateindependent reconstruction ceases to work when the subsystem $D$ becomes larger than $A$. A toy model which mimics this situation is depicted below.

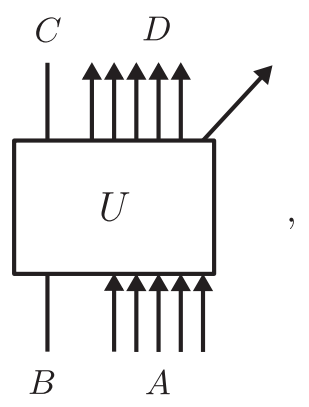

where $|D|>|A|$. Small portion of the outgoing modes will escape to the environment while the rest returns to the black hole. For black holes in a flat space, those returning modes are typically confined in the radiation zone.

Let us examine where $D$ is entangled with. When $U$ is scrambling and $d_{A} \leq d_{D}$, we obtain [7]

$$
I(D, C \bar{A}) \approx 2 \log _{2} d_{A} \quad I(D, \bar{B}) \approx 2\left(\log _{2} d_{D}-\log _{2} d_{A}\right) .
$$

This suggests that there exists $\simeq d_{A}$-dimensional subspace ( $\simeq \log _{2} d_{A}$ qubits) of $D$ which is entangled with $C \bar{A}$. For operators acting on this subspace, entangled partners can be reconstructed on $C \bar{A}$ in a state-independent manner as these are not entangled with $\bar{B}$. However, the remaining $\approx d_{D} / d_{A^{-}}$ dimensional subspace $\left(\simeq \log _{2} d_{D}-\log _{2} d_{A}\right.$ qubits $)$ is entangled with the early radiation $\bar{B}$. Hence, their entangled partners must be reconstructed in a state-dependent manner.

This subtle distinction between state-dependent and state-independent partner operators provides an interesting insight on the emergence of the Page curve in the evaporating black hole. Imagine that the outgoing mode is made of $\log _{2} d_{D}$ qubits and release them one by one to the environment which joins the early radiation $\bar{B}$. When we release the first $\log _{2} d_{A}$ qubits, entanglement entropy between the black hole and the early radiation will increase since those qubits are entangled with $C \bar{A}$ (plus qubits in $D$ which have not been released). On the other hand, the remaining $\log _{2} d_{D}-\log _{2} d_{A}$ qubits are entangled with the early radiation $\bar{B}$, so releasing them will decrease the

\footnotetext{
${ }^{10}$ Strictly speaking, the reconstruction error scales as $O\left(\frac{d_{D}^{2}}{d_{A}^{2}}\right)$, so $A$ needs to contain a few more qubits than $D$. We will ignore this subtlety in the discussions.
}

entanglement entropy between the black hole and the environment, leading to the Page curve behavior. ${ }^{11}$

The smoothness of the horizon requires the interior partners to be state independent and the emergence of the Page curve requires those to be state dependent. In an evaporating black hole, two types of interior operators can coexist. In the firewall problem, we typically focus on situations where state-independent modes are dominant as evaporation process is assumed to be slow.

\section{CRITICAL REMARKS ON PREVIOUS PROPOSALS}

The aforementioned reconstruction of interior partner operators reveals certain fundamental flaws in previous proposals for resolutions of the firewall puzzle, including the $\mathrm{ER}=\mathrm{EPR}$ approach $[9,10]$, the state-dependence approach [11], and the quantum computational complexity approach [31]. These approaches, namely, those bundled as the $\mathrm{ER}=\mathrm{EPR}$ approach, appear to be widely accepted without much scrutiny in the current mainstream research community. In this section, we revisit these previous approaches critically and point out explicitly what went wrong. A more comprehensive set of criticisms are presented in [23].

\section{A. Remarks on $\mathbf{E R}=\mathbf{E P R}$}

Let us begin with proposals, often bundled under the slogan ER = EPR due to Susskind and Maldacena [9]. These proposals roughly go as follows. The distillation of the qubit $R_{D}$ by the outside observer Bob generates perturbations which will become a high-energy density near the horizon as shown in Fig. 5(b). This perturbation spoils the quantum entanglement between $D$ and $\tilde{D}$ and creates a firewall (or Alice may be killed by the shockwave). As such, Alice is not able to observe entanglement between $D$ and $\tilde{D}$. One may draw the Penrose diagram corresponding to these proposals by explicitly treating $R_{D}$ as a gravitational shockwave.

The ER = EPR approach to the firewall puzzle, however, is inconsistent with the implication of the black hole decoupling theorem which suggests that the outgoing mode $D$ is no longer entangled with the early radiation $\bar{B}$. A new partner mode $\tilde{D}$ after perturbation by an infalling observer is distinct from the original partner mode $R_{D}$, and the construction of $\tilde{D}$ does not depend at all on how the black hole was initially entangled with its early radiation $\bar{B}$. Here the observer dependence of the black hole interior plays a key role. The infalling observer will see an interior which is prepared by her own backreaction. Her infalling experience

\footnotetext{
${ }^{11}$ A similar argument for the state dependence in the Page curve derivation has been recently made in [30]. In our understanding, however, we disagree on its implication to the firewall problem.
} 
as well as her black hole interior will not be affected by any quantum operations localized on the early radiation. In fact, whether the black hole is young or old does not matter for crossing the horizon.

Another counterargument against the $\mathrm{ER}=\mathrm{EPR}$ approach is presented in later subsections too.

\section{B. Remarks on state dependence}

Next, let us discuss the state-dependence approach which postulates that interior operators may be dependent on the initial state of the black hole. Such a viewpoint has been promoted largely by Papadodimas and Raju as a potential resolution of the firewall puzzle; see [11] for instance. In the absence of an infalling observer, it is certainly true that interior partner operators depend on the initial state of the black hole. In the presence of an infalling observer, however, the construction of interior operator $\tilde{D}$ dynamically changes and becomes state independent due to the decoupling theorem. Hence, unfortunately the statedependence approach also is not consistent with the fact that the black hole scrambles unitarily.

\section{Remarks on computational complexity}

Finally, let us discuss the quantum circuit or quantum computational complexity approach. The central idea is to accept the violation of the monogamy of entanglement. Yet, by postulating that highly complex interior operators cannot be measured in physically reasonable time scales, one may argue that the violation is essentially not detectable. Ideas along this line were originally proposed by Harlow and Hayden [31]. In the absence of an infalling observer, it is certainly true that interior operators can potentially have high quantum circuit complexity. When the unitary operator $K$ in Eq. (1) is highly complex, the interior partner operator will be also highly complex. In the presence of an infalling observer, however, the complexity of interior operator $\tilde{D}$, which was dynamically created by the gravitational backreaction, does not depend on the initial state of the black hole at all. Furthermore, one can construct $\tilde{D}$ explicitly in an efficient manner as we have already seen in the present paper. Hence, we claim that the quantum complexity approach does not resolve the firewall puzzle. $^{12}$

\section{Remarks on complementarity}

We have discussed why previous proposals, such as the $\mathrm{ER}=\mathrm{EPR}$ approach, fail to resolve the firewall puzzle. The crux of our counterarguments was that these approaches are inconsistent with predictions from the black hole decoupling theorem which holds universally for

\footnotetext{
${ }^{12}$ The quantum circuit complexity of the interior partner mode $\tilde{D}$, in the presence of an infalling observer, has an interesting holographic interpretations on the bulk; see [23].
}

unitarily scrambling dynamics. Advocates of these proposals, however, often argue that such inconsistencies may not be detected from the outside observer and thus one does not need to be bothered by them seriously. Ideas along this line of philosophy originate from the black hole complementarity approach due to Susskind who postulated that an apparent violation of quantum mechanics is acceptable if the violation cannot be verified by a single observer. Here, we demonstrate fundamental flaws in complementaritylike arguments.

Let us briefly recall how the complementaritylike argument would arise in the ER $=$ EPR approach. Based on naive predictions from the bulk geometry, the ER $=E P R$ approach postulates that the infalling observer $A$ from one side will be hit by a shockwave $R_{D}$ from the other side even though two sides are not coupled on the boundaries. This conclusion is of course incorrect as we have already seen in Sec. VI B, but let us tentatively assume that $A$ and $R_{D}$ will encounter in the black hole interior. Since the encounter between $A$ and $R_{D}$ is nonlocal from the perspective of the boundaries, such nonlocal interactions should be hidden from the perspective of the outside observer. One possible argument for avoiding such nonlocality, which will turn out to be incorrect, is based on the following observation. In order for the outside observer to verify whether $A$ and $R_{D}$ have encountered inside the black hole or not, he needs to save $A$ from the black hole interior and pull her back to the black hole exterior. Then he may ask $A$ if she has encountered $R_{D}$ or not. There are a few methods of saving a quantum state, which has fallen into a black hole, and reconstructing it in the black hole exterior. One method is to perform the traversable wormhole protocol [32] or the Hayden-Preskill recovery protocol [18] by coupling two sides of the black hole.

The complementaritylike argument for avoiding the nonlocality then goes roughly as follows. Since saving $A$ from the interior appears to require nonlocal coupling between two boundaries, the nonlocality resulting from the encounter between $A$ and $R_{D}$ cannot be detected unless we actually make two boundaries coupled. Hence, from the perspective of the outside observer, the locality violation will not be detected.

The above speculation is based on a false assumption that saving the infalling observer $A$ from the black hole interior requires coupling two sides. As we have mentioned in Sec. III E, an infalling observer in a two-sided black hole can be saved from the black hole by quantum operations which act solely on one side of the black hole [8]. The rescued observer will tell us that she was not hit by the shockwave $R_{D}$ as the whole procedure is localized only on one side of the black hole and has no dependence on the other side where $R_{D}$ is supported. It is thus incorrect to say that an inconsistency cannot be verified by an outside observer since no inconsistency has occurred. 


\section{MORE ON DECOUPLING THEOREM}

The black hole decoupling theorem is applicable only when the time separation $\Delta t$ between an observer $A$ and the outgoing mode $D$ satisfies $\Delta t \geq t_{\text {scr }}$ and $d_{A} \gtrsim d_{D}$. In this section, we discuss the cases which do not satisfy these two assumptions.

Let us begin with the cases with $\Delta t \leq t_{\mathrm{scr}}$. We present three observations supporting our conclusions concerning the firewall puzzle and the Marolf-Wall puzzle.

(i) Singularity: Let us look at this problem in the twosided AdS black hole where the system is in the TFD (thermofield-double) state. If the time separation $\Delta t$ between $A$ and $D$ is much shorter than the scrambling time $\Delta t \ll t_{\text {scr }}$, the gravitational backreaction from $A$ is negligible. Then, $A$ and $R_{D}$ might appear to encounter inside the black hole as the unperturbed Penrose diagram seems to suggest. However, the trajectories of $A$ and $R_{D}$ will intersect near the singularity [Fig. 5(b)]. It is then not surprising if effective bulk descriptions break down severely there. Also, the encounter location will be outside the region where the Rindler approximation is applicable. As such, we can safely ignore the cases $\Delta t \ll t_{\text {scr. }}$. This observation, however, does not provide a satisfactory justification for intermediate time scales.

(ii) Entanglement quality: If $\Delta t \lesssim t_{\mathrm{scr}}$, an infalling observer will encounter the outgoing mode $D$ away from the horizon. Recall that the proper temperature near the Rindler horizon is given by $T=\frac{1}{2 \pi \rho}$ where $\rho$ is the proper distance from the horizon. As one goes away from the horizon, the density of thermal entropy becomes smaller and the quality of quantum entanglement, which an infalling observer may be able to detect via simple free fall, will deteriorate. Hence, there is no inconsistency even if the decoupling phenomena are weak.

(iii) Measurement strength: Recall that the decoupling phenomena occur as a result of the interaction between the observer $A$ and the outgoing mode $D$, which is quantified by the decay of OTOCs. This suggests that the decoupling, as well as creation of $\tilde{D}$, occurs as much as the observer $A$ measures $D$. Then, even if $D$ and $\tilde{D}$ are not perfectly entangled, there is no contradiction with the monogamy of entanglement since $A$ is observing $D$ (and its partner $\tilde{D}$ ) only weakly.

If $A$ wishes to measure $D$ more directly or strongly, she may increase the size of herself $\left(d_{A}\right)$ to enhance gravitational scattering or perform actual physical measurement (e.g., number operator) on $D$ upon encountering it. We expect that such strong interactions or direct measurements will lead to significant decay of OTOCs and create $\tilde{D}$ which is nearly perfectly entangled with $D$.
Next, let us justify the condition of $d_{A} \gtrsim d_{D}$ by presenting three observations.

(iv) AMPS: For an application to the firewall puzzle, namely, the AMPS thought experiment, we can easily justify this requirement. In the thought experiment, the outside observer distills $R_{D}$ and hand it to the infalling observer $A$ who jumps into the black hole to verify the entanglement between $D$ and $\tilde{D}$. This effectively realizes a situation where $d_{A}=d_{D}^{2}$ with a pure state input on $A$ as discussed in Appendix C.

(v) Metaphysical explanation: In order to experience some physics, the infalling observer $A$ herself should carry some amount of entropies, at least as much as the objects $D$ she is going to measure. While being metaphysical, this explanation can be further justified from the aforementioned observation (iii) which suggests the decoupling occurs as much as the observer measured $D$.

(vi) Effective QFT: One may interpret $A$ and $D$ as infalling and outgoing modes of low energy QFT on the lhs wedge, respectively, while $B$ and $C$ can be viewed as all the other high-energy degrees of freedom (d.o.f) associated with the future and past horizons, respectively. It is thus natural to assume $d_{A}=d_{D}$ as also discussed in Sec. VIC. Here $A$ and $D$ are d.o.f which an outside observer can easily see.

On the other hand, the infalling observer, who travels nearly at the speed of light, will propagate along the infalling mode $A$. Hence, it is natural to expect that the initial state of $A$ may become irrelevant as long as the initial state is chosen from low energy subspace of the QFT. Namely, regardless of the initial state of $A$, she will see the same bulk effective QFT description near the horizon. This is indeed the case as the creation of $\tilde{D}$ occurs for any initial state of $A$. However, it is worth emphasizing that the map from the bulk QFT to the boundary is dependent on the initial state of $A$. Here the infalling observer will have an access to the outdoing mode $D$ and the interior mode $\tilde{D}$ instead of $A$.

We have presented justifications of our conclusions for the cases where the system does not satisfy underlying assumptions of the decoupling theorem. Perhaps a better question to ask is whether we actually need to include the infalling observer as additional degrees of freedom or not in deriving the bulk description from the boundary description. From the outside viewpoint, the infalling and outgoing modes influence each other in a dynamical manner. When switching to the infalling observer's viewpoint, infalling modes somehow decohere, perhaps due to the choice of the infalling trajectory. Ultimately, we hope to understand why the interior operator is "automatically decoded" simply by switching to the infalling observer's frame. 


\section{ACKNOWLEDGMENTS}

I thank Yoni BenTov and Yingfei Gu for discussions. Research at the Perimeter Institute was supported by the Government of Canada through Innovation, Science and Economic Development Canada and by the Province of Ontario through the Ministry of Economic Development, Job Creation and Trade.

\section{APPENDIX A: REVIEW OF QUANTUM INFORMATION CONCEPTS}

\section{Scrambling}

Here we recall the definition of scrambling in terms of OTOCs.

At infinite temperature, OTOCs are defined with respect to the maximally mixed state $\rho=\frac{1}{d} \mathbb{I}$,

$$
\langle W(t) Z(0) Y(t) X(0)\rangle \equiv \frac{1}{d} \operatorname{Tr}[W(t) Z(0) Y(t) X(0)],
$$

where time-evolved operators are defined by $O(t)=$ $U^{\dagger} O(0) U$. The phenomenon of scrambling is often associated with the decay (decorrelation) of OTOCs from their initial values when $W, Z, Y, X$ are basis operators such as Pauli and Majorana operators. In a more genetic language, the system is said to be scrambling at time $t$ if the following asymptotic decomposition holds for all the local operators $W, Z, Y, X[18]:$

$$
\begin{aligned}
& \langle W(t) Z(0) Y(t) X(0)\rangle \\
& \approx\langle W Y\rangle\langle Z\rangle\langle X\rangle+\langle Z X\rangle\langle W\rangle\langle Y\rangle-\langle W\rangle\langle Z\rangle\langle Y\rangle\langle X\rangle,
\end{aligned}
$$

where expectation values are defined by $\langle O\rangle \equiv \frac{1}{d} \operatorname{Tr}[O]$.

We immediately see that the asymptotic value for a large system size is zero when $X, Y, Z, W$ are traceless. This asymptotic form has been derived for Haar random unitary [33] and from the eigtenstate hermalization hypothesis (ETH) [34]. Corrections to the asymptotic form are polynomially (exponentially) suppressed with respect to the system size $n$ for systems with (without) conserved quantities.

At finite temperature, OTOCs are defined with respect to a thermal mixed state $\rho=e^{-\beta H} / \operatorname{Tr}\left(e^{-\beta H}\right)$,

$$
\langle W(t) Z(0) Y(t) X(0)\rangle \equiv \operatorname{Tr}\left[\rho^{\alpha} W(t) \rho^{\beta} Z(0) \rho^{\gamma} Y(t) \rho^{\delta} X(0)\right],
$$

where

$$
\alpha, \beta, \gamma, \delta \geq 0 \quad \alpha+\beta+\gamma+\delta=1 .
$$

Specifics of $\alpha, \beta, \gamma, \delta$ do not affect the main result, and we will focus on $\alpha=\beta=\gamma=\delta=1 / 4$ unless otherwise stated. We can infer the asymptotic form of finite temperature OTOCs in an analogous manner by using thermal expectation values,

$$
\begin{gathered}
\langle O\rangle \equiv \operatorname{Tr}(\rho O) \quad\left\langle O_{1} O_{2}\right\rangle \equiv \operatorname{Tr}\left(\rho^{\alpha} O_{1} \rho^{\beta} O_{2}\right) \\
\alpha, \beta>0 \quad \alpha+\beta=1 .
\end{gathered}
$$

Again, specifics of $\alpha, \beta$ does not affect the main result, and we will choose $\alpha=\beta=1 / 2$. The asymptotic form of OTOCs at finite temperature can be also derived from ETH [35].

Let us present some intuition behind the aforementioned definition of scrambling. The thermal ensemble $\rho$ has significant contributions from eigenstates within typical energy subspace at given temperature. Hence, one may interpret each insertion of powers of $\rho$ as projection onto typical energy subspace. The asymptotic form of OTOC suggests that the time evolution $U$ resembles Haar random unitary within the typical energy subspace. Here we are particularly interested in operators $X, Y, Z, W$ which do not change the energy of the system much so that the system remains within the typical energy subspace.

It is worth emphasizing that our main focus is on systems after the scrambling time when OTOCs have decayed to asymptotic values. The famous Lyapunov behavior is typically observed only before the scrambling time.

\section{Diagrams}

In this paper, we extensively use diagrammatic tensor notations in order to express wave functions and operators as well as physical processes. While these techniques have become standard in the past several years, it would be still beneficial to have a brief summary to make the presentation self-consistent. Wave functions and operators are represented by

$$
|\psi\rangle=\frac{\uparrow}{T} \quad\langle\psi|=\frac{T^{*}}{\downarrow} \quad O=\frac{\hat{T}_{\text {out }}}{\boldsymbol{T}_{\text {in }}},
$$

which can be also explicitly written as

$$
|\psi\rangle=\sum_{j} T_{j}|j\rangle \quad\langle\psi|=\sum_{j} T_{j}^{*}\left\langle j\left|\quad O=\sum_{i j} O_{i j}\right| i\right\rangle\langle j| .
$$

By using these tensors as building blocks, one can express various physical processes in a graphical manner. For instance, an expectation value can be represented by 


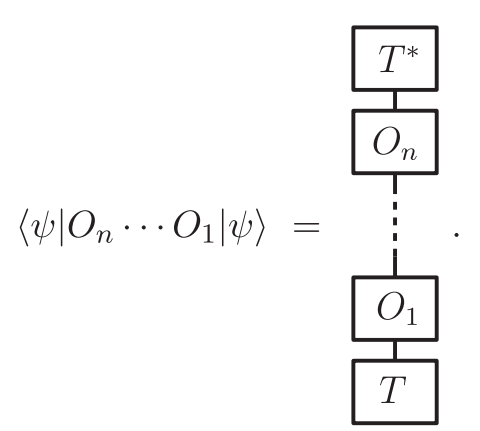

(A8)

In order to associate a physical process to an equation like $\left\langle\psi\left|O_{n} \cdots O_{1}\right| \psi\right\rangle$, one needs to read it from the right to the left, i.e., the initial state $|\psi\rangle$ is acted by $O_{1}, O_{2}, \cdots$ sequentially and then is projected onto $|\psi\rangle$. In the diagrammatic notation, one needs to read the figure from the bottom to the top, i.e., the time flows upward in the diagram.

Consider a Hilbert space $\mathcal{H}_{R}=\mathbb{C}^{d_{R}}$ whose dimensionality is $d_{R} \equiv\left|\mathcal{H}_{R}\right|$. An identity operator, $I_{R}=\sum_{j=1}^{d_{R}}|j\rangle\langle j|$, can be expressed as a straight line (i.e., a trivial tensor) since its inputs and outputs are the same,

$$
I_{R}=\frac{\uparrow}{\Psi_{\uparrow}}=\overbrace{\text { in }}^{\text {out }} .
$$

This diagram has one input leg (index) and one output leg. One may bend the line and construct the following diagram:

$$
|\mathrm{EPR}\rangle_{R \bar{R}} \propto \sum_{j=1}^{d_{R}}|j\rangle \otimes|j\rangle=\stackrel{R}{\mathrm{4}} \stackrel{\bar{R}}{ },
$$

which is the same trivial line, but with two output legs instead of one in and one out. This diagram represents an unnormalized EPR pair defined on $\mathcal{H}_{R} \otimes \mathcal{H}_{\bar{R}}$.

We utilize the following notation for normalized EPR pairs:

$$
\left.|\mathrm{EPR}\rangle_{R \bar{R}}=\frac{1}{\sqrt{d_{R}}} \sum_{j=1}^{d_{R}}|j\rangle \otimes|j\rangle=\stackrel{R}{\square}\right]^{\bar{R}},
$$

where the black dot carries a factor of $1 / \sqrt{d_{R}}$.

$$
\text { 3. } O, O^{\dagger}, O^{T}, O^{*}
$$

It is also beneficial to define $O, O^{\dagger}, O^{T}, O^{*}$ and review some useful relations associated with them. Given an operator $O$, the complex-conjugate transpose $\mathrm{O}^{\dagger}$, the transpose $O^{T}$, and the complex-conjugate are defined by

$$
\begin{aligned}
& O=\sum_{i, j} O_{i j}|i\rangle\left\langle j\left|\quad O^{\dagger}=\sum_{i, j} O_{i j}^{*}\right| j\right\rangle\langle i| \\
& O^{T}=\sum_{i, j} O_{i j}|j\rangle\left\langle i\left|\quad O^{*}=\sum_{i, j} O_{i j}^{*}\right| i\right\rangle\langle j|,
\end{aligned}
$$

where $O^{\dagger}=\left(O^{T}\right)^{*}$. In the tensor diagrams, the transpose can be represented as follows:

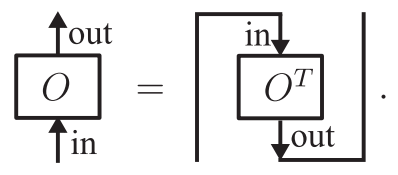

The following relation is particularly useful in constructing partner operators:

$$
(O \otimes I)|\mathrm{EPR}\rangle=\left(I \otimes O^{T}\right)|\mathrm{EPR}\rangle,
$$

or graphically

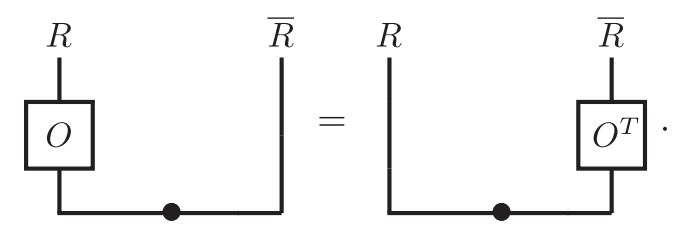

\section{State representation}

Given an operator $O$ acting on a Hilbert space $\mathcal{H}$, its state representation (Choi representation), supported on $\mathcal{H} \otimes \mathcal{H}$, is defined by

$$
|O\rangle \equiv(O \otimes I)|\mathrm{EPR}\rangle=\stackrel{O}{O} .
$$

In this regard, the EPR pair is a state representation of an identity operator; $|\mathrm{EPR}\rangle=|I\rangle$. See [17] for further discussions on the state representation.

Given a density matrix $\rho_{R}$ on $\mathcal{H}_{R}$, consider a purification (thermofield double state) on $\mathcal{H}_{R} \otimes \mathcal{H}_{\bar{R}}$,

$$
\rho=\sum_{j} p_{j}\left|\psi_{j}\right\rangle\left\langle\psi_{j}\left|\rightarrow \sum_{j} \sqrt{p_{j}}\right| \psi_{j}\right\rangle \otimes\left|\psi_{j}^{*}\right\rangle
$$

The purified state can be viewed as a state representation of $\rho_{R}$. By introducing an unnormalized state

$$
\tilde{\rho}_{R} \equiv d_{R} \rho_{R} \quad \operatorname{Tr}\left[\tilde{\rho}_{R}\right]=d_{R}
$$


we have

$$
\left|\tilde{\rho}_{R}^{1 / 2}\right\rangle=\sum_{j} \sqrt{p_{j}}\left|\psi_{j}\right\rangle \otimes\left|\psi_{j}^{*}\right\rangle
$$

\section{APPENDIX B: STATEMENT OF DECOUPLING THEOREM}

Below we sketch a proof of $\rho_{\bar{B} D} \approx \rho_{\bar{B}} \otimes \rho_{D}$. When two states are close in $L^{1}$ distance, they cannot be distinguished by any measurement. The strategy is to show that $\rho_{\bar{B} D}$ is close to a maximally mixed state $\frac{1}{d_{\bar{B}} d_{D}} I_{B} \otimes I_{D}$ in the $L^{1}$ distance. More precisely, we will prove the following bound:

$$
\left\|\rho_{\bar{B} D}-\frac{1}{d_{\bar{B}} d_{D}} I_{B} \otimes I_{D}\right\|_{1}^{2} \leq O\left(\frac{d_{D}^{2}}{d_{A}^{2}}\right)+O(\epsilon),
$$

where the $L^{1}$ norm is defined by $\|O\|_{1}=\operatorname{Tr} \sqrt{O^{\dagger} O}$. Here, $\epsilon$ is the error from the asymptotic form of OTOCs which is typically suppressed polynomially in the system size $n$.

The derivation is straightforward. Observe

$$
\begin{gathered}
\left\|\rho_{\bar{B} D}-\frac{1}{d_{\bar{B}} d_{D}} I_{B} \otimes I_{D}\right\|_{1}^{2} \leq d_{\bar{B}} d_{D} \| \rho_{\bar{B} D} \\
-\frac{1}{d_{\bar{B}} d_{D}} I_{B} \otimes I_{D} \|_{2}^{2}=\operatorname{Tr}\left(\rho_{\bar{B} D}^{2}\right) d_{\bar{B}} d_{D}-1 .
\end{gathered}
$$

The rhs can be evaluated by noticing

$$
\operatorname{Tr}\left(\rho_{\bar{B} D}^{2}\right)=\frac{d_{D}}{d_{\bar{B}}} \Delta
$$

Using the OTOC asymptotics, we obtain the claimed bound.

A similar bound can be derived for the cases with a pure state input by analogous analysis. We obtain

$$
\left\|\rho_{\bar{B} D}-\frac{1}{d_{\bar{B}} d_{D}} I_{B} \otimes I_{D}\right\|_{1}^{2} \leq O\left(\frac{d_{D}^{2}}{d_{A}}\right)+O(\epsilon) .
$$

For a finite temperature generalization of the decoupling theorem, see [18] and the Appendix in [36].

\section{APPENDIX C: MORE ON AMPS PUZZLE}

Consider an old black hole that is maximally entangled with the early radiation. For simplicity of discussion, we assume that the initial state is EPR pairs. Assume that the black hole time evolves by some unitary dynamics $U$. Consider a scenario where an outside observer distills some qubits $R_{D}$ that would be entangled with the outgoing mode $D$ by applying some distillation unitary operator $\mathcal{D}$,

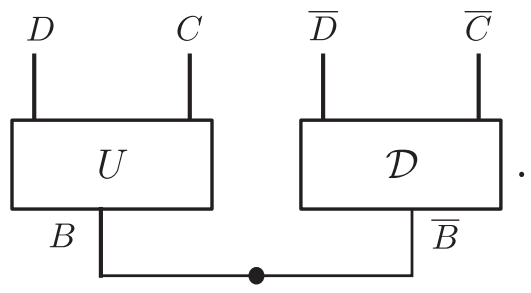

A natural choice of $\mathcal{D}$ is to take $\mathcal{D}=U^{*}$ so that $D$ and $\bar{D}$ form EPR pairs, but we will keep it unspecified.

Imagine that the outside observer hands these distilled qubits to the infalling observer who is going to jump into a black hole. After the time evolution, we will obtain the following quantum state:

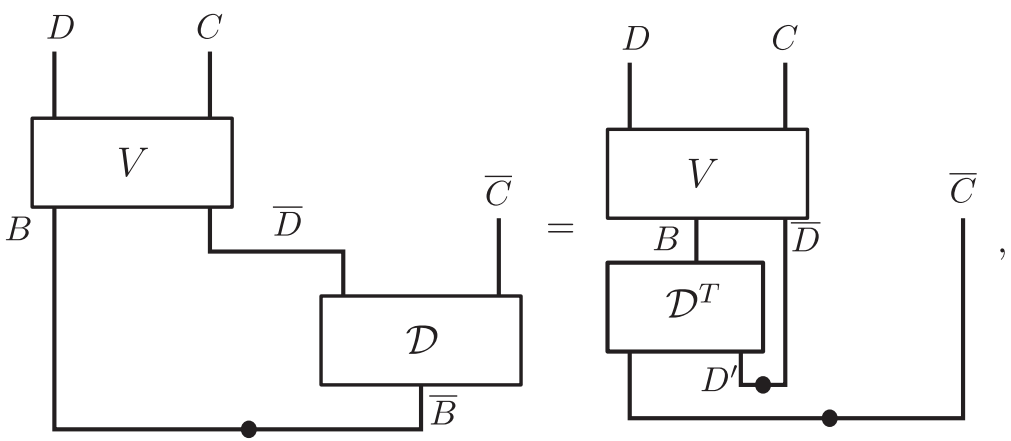

where the black hole time evolves by some unitary dynamics $V$. Here $U$ and $V$ are different unitary operators in general as the size of the Hilbert space changed by adding the distilled qubits to the black hole. By viewing
$V\left(\mathcal{D}^{T} \otimes I_{\bar{D}}\right)$ as the total dynamics of the black hole, the above diagram corresponds to the interior reconstruction problem with the infalling observer being prepared in EPR pairs on $D^{\prime} \bar{D}$. As long as $V\left(\mathcal{D}^{T} \otimes I_{\bar{D}}\right)$ is scrambling, one 
can construct interior partner operators on the remaining black hole $C$. We expect that this is indeed the case for generic choices of $\mathcal{D} .^{13}$
APPENDIX D: FINITE TEMPEARTURE

We construct partner operators at finite temperature. We consider the following quantum state at finite temperature:

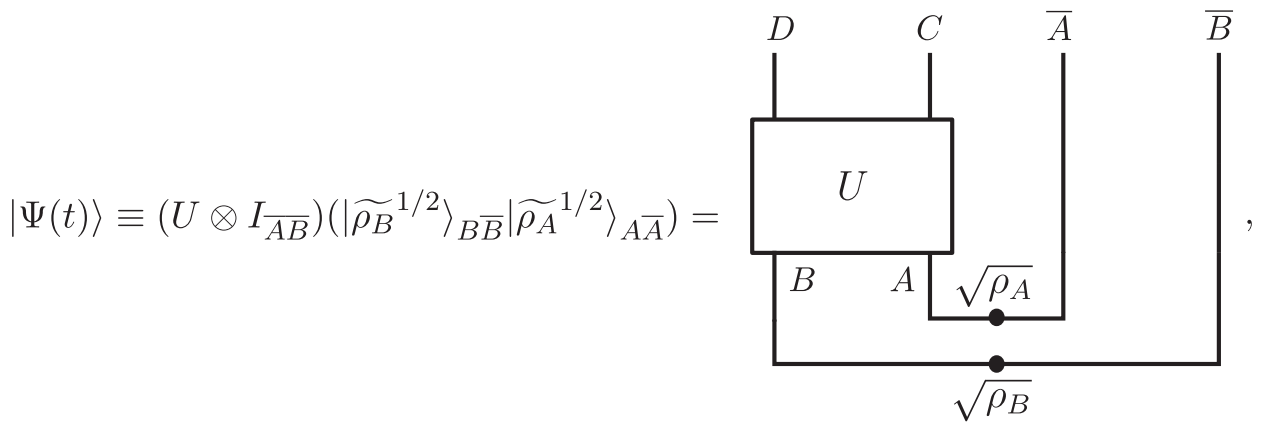

where the black hole was initially in the thermofield double state $\left|{\widetilde{\rho_{B}}}^{1 / 2}\right\rangle$ on $B \bar{B}$ and then was perturbed by the measurement probe prepared in some entangled state $\left|\widetilde{\rho}_{A}^{1 / 2}\right\rangle$.

The construction procedure be applied to finite temperature systems which satisfy a certain technical assumption [18]. Given the input ensemble $\rho_{A B}=\rho_{A} \otimes \rho_{B}$, we will assume that the output ensemble factors into $C$ and $D$,

$$
\rho_{C D} \equiv U \rho_{A B} U^{\dagger} \approx \rho_{C} \otimes \rho_{D}
$$

Unfortunately, this condition is problematic for physical reasons; if $\rho_{C D}$ is thermal, $C$ and $D$ are entangled. We will present a justification for such an assumption in Appendix F. For our purpose, it suffices to coarse-grain and remove preexisting correlations between $C$ and $D$ in a thermal ensemble $\rho_{C D}$.

\section{Distillation}

Having imposed the aforementioned assumption, generalization to finite-temperature systems is straightforward; we simply replace all the dots in the previous diagrammatic calculations with $\sqrt{\rho_{R}}$. One subtlety is that, since the outgoing mode $D$ is thermal, partner operators $\Omega\left(O_{D}\right)$ must satisfy

$$
\left\langle\Psi(t)\left|O_{D}^{\dagger} \otimes \Omega\left(O_{D}\right)\right| \Psi(t)\right\rangle \approx\left\langle O_{D}^{\dagger} O_{D}\right\rangle \equiv \operatorname{Tr}\left[\rho_{D}^{1 / 2} O_{D}^{\dagger} \rho_{D}^{1 / 2} O_{D}\right],
$$

reproducing thermal expectation values. This relation can be viewed as the definition of interior partner operators for finite temperature systems.

Hence we consider distillation protocol of $\left|{\widetilde{\rho_{D}}}^{1 / 2}\right\rangle$ from $D$ and $C \bar{A}$, which proceeds in the following manner:

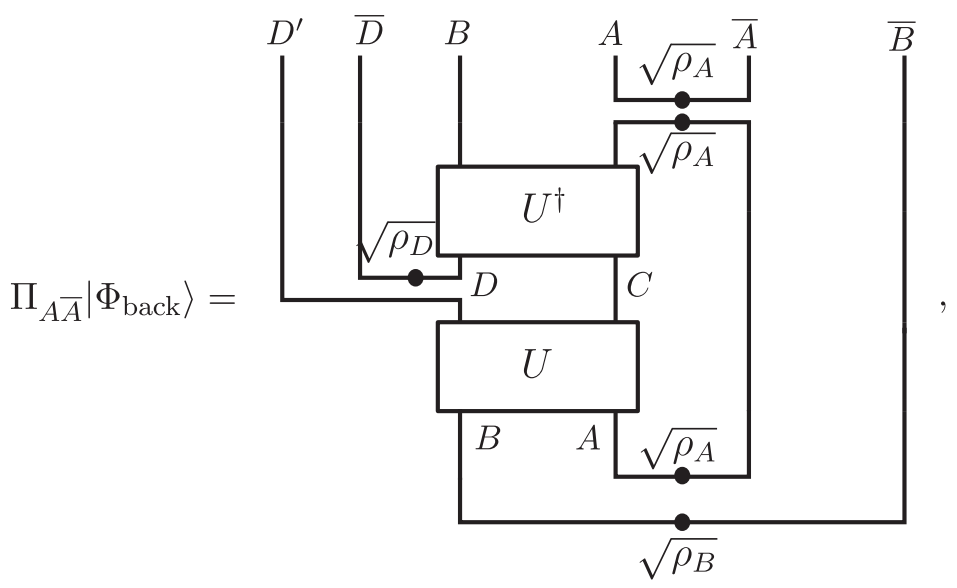

\footnotetext{
${ }^{13}$ Whether there exist special choices of $\mathcal{D}$ which make $V\left(\mathcal{D}^{T} \otimes I_{\bar{D}}\right)$ nonscrambling or not is an interesting question. If such $\mathcal{D}$ exists, it would be performing the Hayden-Preskill recovery which would protect an infalling observer from crossing the horizon. See [8] for details.
} 
where $\left|{\widetilde{\rho_{D}}}^{1 / 2}\right\rangle$ is inserted on $\bar{D} D$ and projection $\Pi_{A \bar{A}} \equiv$ $\left|{\widetilde{\rho_{A}}}^{1 / 2}\right\rangle\left\langle{\widetilde{\rho_{A}}}^{1 / 2}\right|$ is applied on $A \bar{A}$.

The probability amplitude to measure $\left|{\widetilde{\rho_{A}}}^{1 / 2}\right\rangle$ can be evaluated via the asymptotic form of OTOCs at finite temperature. After some calculations (see Appendix E), we obtain

$$
\Delta \equiv\left\langle\Phi_{\text {back }}\left|\Pi_{A \bar{A}}\right| \Phi_{\text {back }}\right\rangle \approx \operatorname{Tr}\left[\rho_{D}^{3}\right]
$$

for $d_{A} \gg d_{D}{ }^{14}$ The following lower bound is useful:

$$
\begin{aligned}
& \left\langle\Phi_{\text {back }}\left|\Pi_{D^{\prime} \bar{D}} \Pi_{A \bar{A}}\right| \Phi_{\text {back }}\right\rangle \\
& \quad \geq\left\langle\Phi_{\text {back }}\left|\Pi_{D^{\prime} \bar{D}} \Pi_{A \bar{A}} \Pi_{B \bar{B}}\right| \Phi_{\text {back }}\right\rangle=\operatorname{Tr}\left[\rho_{D}^{2}\right]^{2} .
\end{aligned}
$$

Hence, the probability amplitude to measure $\left|{\widetilde{\rho_{D}}}^{1 / 2}\right\rangle$ after the postselection is lower bounded by

$\frac{1}{\Delta}\left\langle\Phi_{\text {back }}\left|\Pi_{D^{\prime} \bar{D}} \Pi_{A \bar{A}}\right| \Phi_{\text {back }}\right\rangle \gtrsim \frac{\operatorname{Tr}\left[\rho_{D}^{2}\right]^{2}}{\operatorname{Tr}\left[\rho_{D}^{3}\right]}=2^{2\left(S_{D}^{(3)}-S_{D}^{(2)}\right)}$,

where $S_{D}^{(\alpha)}$ is the Rényi- $\alpha$ entropy of $\rho_{D}$. If $S_{D}^{(3)}-S_{D}^{(2)} \approx 0$, $\left|\widetilde{\rho_{D}}{ }^{1 / 2}\right\rangle$ can be distilled on $D^{\prime} \bar{D}$.

\section{Dressed operator growth}

The distillation procedure constructs the following interior partner operators:
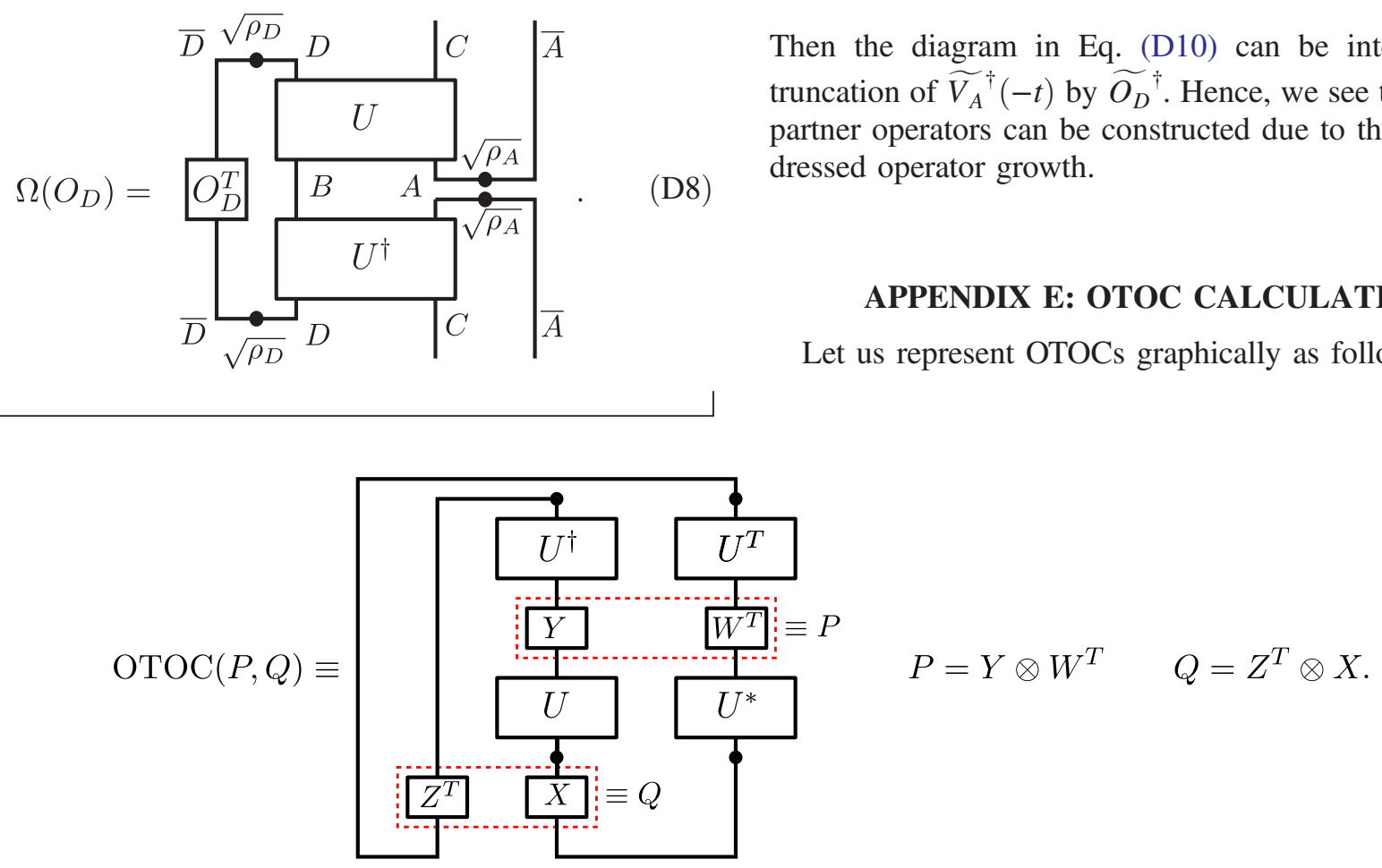

\footnotetext{
${ }^{14}$ This is the result for $\alpha=\beta=\gamma=\delta=1 / 4$ in Eq. (A3). When $\alpha=1$, we obtain $\Delta \approx \operatorname{Tr}\left[\rho_{D}^{3}\right] \operatorname{Tr}\left[\rho_{A}^{3 / 2}\right] \operatorname{Tr}\left[\rho_{A}^{1 / 2}\right]$. If the variance of the spectrum of $\rho_{A}$, normalized by its mean, approaches zero asymptotically, $\operatorname{Tr}\left[\rho_{A}^{3 / 2}\right] \operatorname{Tr}\left[\rho_{A}^{1 / 2}\right] \approx 1$.
} 
Due to linearity, we can generalize the above OTOCs as a function of $P, Q$ which act on $\mathcal{H}^{\otimes 2}$. We then have

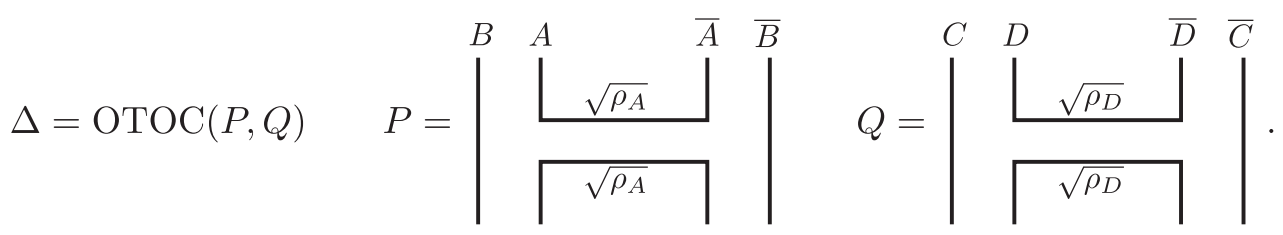

Using the OTOC asymptotics, we obtain

$$
\Delta=\operatorname{Tr}\left(\rho_{D}^{3}\right)+\operatorname{Tr}\left(\rho_{A}^{3}\right)-\operatorname{Tr}\left(\rho_{D}^{3}\right) \operatorname{Tr}\left(\rho_{A}^{3}\right) \approx \operatorname{Tr}\left(\rho_{D}^{3}\right) .
$$

\section{APPENDIX F: ON $\rho_{C D} \approx \rho_{C} \otimes \rho_{D}$}

Here we present a justification of the assumption $\rho_{C D} \approx \rho_{C} \otimes \rho_{D}{ }^{15}$

\section{Coarse-graining}

For simplicity of discussion, we approximate $\rho_{C D}$ as a thermal ensemble $\rho_{C D}=\frac{e^{-\beta H_{C D}}}{\operatorname{Tr}\left[e^{\left.-\beta H_{C D}\right]}\right.}$. Consider the thermofield double state $\left|{\widetilde{\rho_{C D}}}^{1 / 2}\right\rangle$ supported on $C, D, \bar{C}, \bar{D}$. The assumption $\rho_{C D} \approx \rho_{C} \otimes \rho_{D}$ asserts that $C(D)$ is entangled only with $\bar{C}(\bar{D})$, respectively. In general, however, this is not the case.

Here we are interested in what degrees of freedom the outgoing mode $D$ is entangled with. Eigenstates of the Hamiltonian $H_{C D}$ are entangled between $C$ and $D$. At low temperature, a fewer number of states contribute to the thermal ensemble and thus entanglement between $C$ and $D$ survives in $\rho_{C D}$. Then the "partner" operators of $D$ can be found on $C$ due to the preexisting entanglement in $\rho_{C D}$. In this case, the system does not need to be perturbed in order to construct the partner operator from the beginning. Indeed, such modes on $D$ do not lead to the firewall puzzle since, in order for the puzzle to exist, $D$ needs to be entangled with $\overline{C D}$ in the first place. See Fig. 7.

There is another reason why entanglement between $C$ and $D$ may not be an interesting one to focus on. In discrete spin systems, entanglement between $C$ and $D$ is mostly of short-range nature. In quantum field theories, such entanglement is UV divergent and hence is cutof-dependent property which should be removed by some proper coarsegraining. We may simply discard short-range entanglement between $C$ and $D$ and construct subspace $C_{\text {sub }}$ and $D_{\text {sub }}$ on which we may have a factor structure $\rho_{C_{\text {sub }}} \otimes \rho_{D_{\text {sub }}}$. Due to the preexisting entanglement between $C$ and $D$, releasing $D$ to the environment appears to increase entanglement

\footnotetext{
${ }^{15}$ I thank Alexei Kitaev for discussions on this.
}

entropy of the black hole. These UV divergent pieces, however, are often ignored in the Bekenstein bound [38].

\section{Soft mode}

Even if we remove short-range entanglement between $C$ and $D$; however, there still remains some classical correlation between $C$ and $D$ due to the presence of conserved quantities (such as the energy, charge, and angular momentum). The Hayden-Preskill recovery problem for systems with conserved quantities (symmetries) has been discussed in [7]. Here it is convenient to consider two types of physical modes (hard and soft modes) on $C, D$. Roughly, hard modes are those which are associated with change of conserved quantities on $C, D$, whereas soft modes are those which do not change them. It is convenient to imagine an approximate decomposition of the Hilbert space into a block-diagonal form: $\mathcal{H} \approx \bigoplus_{\alpha} \mathcal{H}_{C}^{\alpha} \otimes \mathcal{H}_{D}^{\alpha}$ where $\alpha$ labels the conserved quantity. Soft mode operators are block diagonal, while hard mode operators may change $\alpha$-labels by acting on both $C$ and $D$. Here the thermal density matrix $\rho_{\beta}$ can be interpreted as an approximate projection onto states within a typical energy window. Such a subspace corresponds to some single label of $\alpha$ in the above decomposition, and soft mode operators do not change the energy of the system much. Examples of soft modes include gravitational shockwaves which only shift the horizon, commuting with $\rho_{\beta}$. When $\alpha$-label in $C$ changes, $\alpha$-label in $D$ must change too due to the conservation law. This suggests that hard modes on $D$ are correlated classically with hard modes on $C$. Hence we can focus on entanglement associated with soft modes only. In [7], it has been shown that soft modes on $D$ can be reconstructed by using the Hayden-Preskill recovery protocols.

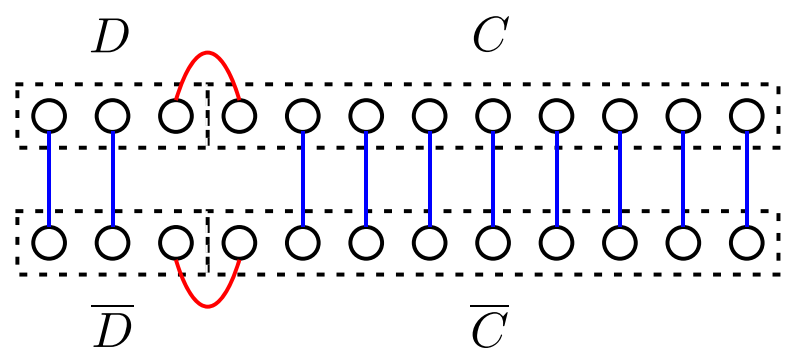

FIG. 7. Short-range entanglement between $C$ and $D$. 
[1] A. Almheiri, X. Dong, and D. Harlow, Bulk locality and quantum error correction in AdS/CFT, J. High Energy Phys. 04 (2015) 163.

[2] F. Pastawski, B. Yoshida, D. Harlow, and J. Preskill, Holographic quantum error-correcting codes: Toy models for the bulk/boundary correspondence, J. High Energy Phys. 06 (2015) 149.

[3] X. Dong, D. Harlow, and A. C. Wall, Reconstruction of Bulk Operators Within the Entanglement Wedge in GaugeGravity Duality, Phys. Rev. Lett. 117, 021601 (2016).

[4] D. L. Jafferis, A. Lewkowycz, J. Maldacena, and S. J. Suh, Relative entropy equals bulk relative entropy, J. High Energy Phys. 06 (2016) 004.

[5] A. Hamilton, D. Kabat, G. Lifschytz, and D. A. Lowe, Holographic representation of local bulk operators, Phys. Rev. D 74, 066009 (2006).

[6] D. Harlow, Tasi lectures on the emergence of the bulk in AdS/CFT, Proc. Sci., TASI2017 (2018) 002.

[7] B. Yoshida, Soft mode and interior operator in the HaydenPreskill thought experiment, Phys. Rev. D 100, 086001 (2019).

[8] B. Yoshida, Firewalls vs. scrambling, J. High Energy Phys. 10 (2019) 132.

[9] J. Maldacena and L. Susskind, Cool horizons for entangled black holes, Fortschr. Phys. 61, 781 (2013).

[10] M. Van Raamsdonk, Evaporating firewalls, J. High Energy Phys. 11 (2014) 038.

[11] K. Papadodimas and S. Raju, An infalling observer in AdS/CFT, J. High Energy Phys. 10 (2013) 212.

[12] A. Almheiri, D. Marolf, J. Polchinski, and J. Sully, Black holes: Complementarity or firewalls?, J. High Energy Phys. 02 (2013) 062.

[13] E. Verlinde and H. Verlinde, Black hole entanglement and quantum error correction, J. High Energy Phys. 10 (2013) 107.

[14] D. A. Roberts, D. Stanford, and L. Susskind, Localized shocks, J. High Energy Phys. 03 (2015) 51.

[15] P. Hayden and J. Preskill, Black holes as mirrors: Quantum information in random subsystems, J. High Energy Phys. 09 (2007) 120.

[16] S. H. Shenker and D. Stanford, Black holes and the butterfly effect, J. High Energy Phys. 03 (2014) 67.

[17] P. Hosur, X.-L. Qi, D. A. Roberts, and B. Yoshida, Chaos in quantum channels, J. High Energy Phys. 02 (2016) 004.

[18] B. Yoshida and A. Kitaev, Efficient decoding for the Hayden-Preskill protocol, arXiv:1710.03363.

[19] D. Marolf and J. Polchinski, Gauge-Gravity Duality and the Black Hole Interior, Phys. Rev. Lett. 111, 171301 (2013).
[20] A. Almheiri, D. Marolf, J. Polchinski, D. Stanford, and J. Sully, An apologia for firewalls, J. High Energy Phys. 09 (2013) 018.

[21] Q. Zhuang, T. Schuster, B. Yoshida, and N. Y. Yao, Scrambling and complexity in phase space, Phys. Rev. A 99, 062334 (2019).

[22] D. A. Roberts, D. Stanford, and A. Streicher, Operator growth in the SYK model, J. High Energy Phys. 06 (2018) 122.

[23] B. Yoshida, Remarks on black hole complexity puzzle, J. High Energy Phys. 10 (2020) 103.

[24] S. D. Mathur, The information paradox: A pedagogical introduction, Classical Quantum Gravity 26, 224001 (2009).

[25] S. L. Braunstein, S. Pirandola, and K. Życzkowski, Better Late Than Never: Information Retrieval from Black Holes, Phys. Rev. Lett. 110, 101301 (2013).

[26] D. Marolf and A. C. Wall, Eternal black holes and superselection in AdS/CFT, Classical Quantum Gravity 30, 025001 (2013).

[27] D. Harlow, Jerusalem lectures on black holes and quantum information, Rev. Mod. Phys. 88, 015002 (2016).

[28] D. Harlow, Wormholes, emergent gauge fields, and the weak gravity conjecture, J. High Energy Phys. 01 (2016) 122.

[29] D. N. Page, Information in Black Hole Radiation, Phys. Rev. Lett. 71, 3743 (1993).

[30] G. Penington, Entanglement wedge reconstruction and the information paradox, J. High Energy Phys. 09 (2020) 002.

[31] D. Harlow and P. Hayden, Quantum computation vs. firewalls, J. High Energy Phys. 06 (2013) 085.

[32] P. Gao, D. L. Jafferis, and A. C. Wall, Traversable wormholes via a double trace deformation, J. High Energy Phys. 12 (2017) 151.

[33] D. A. Roberts and B. Yoshida, Chaos and complexity by design, J. High Energy Phys. 04 (2017) 121.

[34] Y. Huang, F. G. S. L. Brandão, and Y.-L. Zhang, Finite-Size Scaling of Out-of-Time-Ordered Correlators at Late Times, Phys. Rev. Lett. 123, 010601 (2019).

[35] A. Alhambra and J. Riddell (private communication).

[36] B. Yoshida and N. Y. Yao, Disentangling Scrambling and Decoherence Via Quantum Teleportation, Phys. Rev. X 9, 011006 (2019).

[37] X.-L. Qi and A. Streicher, Quantum epidemiology: Operator growth, thermal effects, and SYK, J. High Energy Phys. 08 (2019) 012.

[38] H. Casini, Relative entropy and the Bekenstein bound, Classical Quantum Gravity 25, 205021 (2008). 